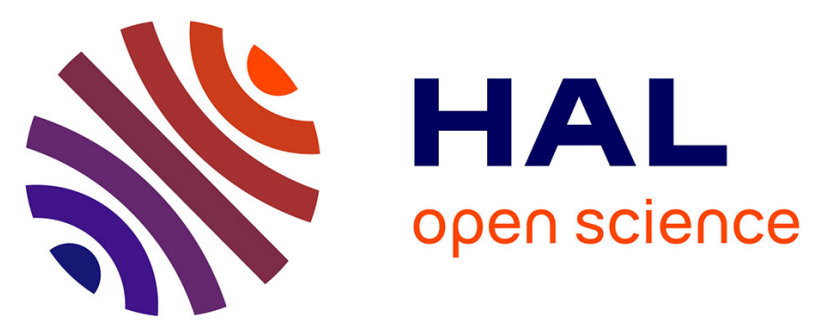

\title{
Glioblastoma Therapy in the Age of Molecular Medicine
} Luiz Henrique Medeiros Geraldo, Celina Garcia, Anna Carolina Carvalho da Fonseca, Luiz Gustavo Feijó Dubois, Tânia Cristina Leite de Sampaio E Spohr, Diana Matias, Eduardo Sabino de Camargo Magalhães, Rackele Ferreira Do Amaral, Barbara Gomes da Rosa, Izabella Grimaldi, et al.

\section{To cite this version:}

Luiz Henrique Medeiros Geraldo, Celina Garcia, Anna Carolina Carvalho da Fonseca, Luiz Gustavo Feijó Dubois, Tânia Cristina Leite de Sampaio E Spohr, et al.. Glioblastoma Therapy in the Age of Molecular Medicine. Trends in Cancer, 2019, 5, pp.46 - 65. 10.1016/j.trecan.2018.11.002 . hal03484495

\section{HAL Id: hal-03484495 https://hal.science/hal-03484495}

Submitted on 20 Dec 2021

HAL is a multi-disciplinary open access archive for the deposit and dissemination of scientific research documents, whether they are published or not. The documents may come from teaching and research institutions in France or abroad, or from public or private research centers.
L'archive ouverte pluridisciplinaire HAL, est destinée au dépôt et à la diffusion de documents scientifiques de niveau recherche, publiés ou non, émanant des établissements d'enseignement et de recherche français ou étrangers, des laboratoires publics ou privés.

\section{(c) (1) $\$$}

Distributed under a Creative Commons Attribution - NonCommerciall 4.0 International 


\section{Glioblastoma therapy in the age of molecular medicine}

Luiz Henrique Medeiros Geraldo ${ }^{\text {a\#* }}$, Celina Garcia ${ }^{a}$, Anna Carolina Carvalho da Fonseca ${ }^{\text {a }}$, Luiz Gustavo Feijó Dubois ${ }^{\mathrm{b}}$, Tânia Cristina Leite de Sampaio e Spohr ${ }^{\mathrm{b}}$, Diana Matias ${ }^{\mathrm{a}, \mathrm{b}}$, Eduardo Sabino de Camargo Magalhães ${ }^{\mathrm{a}}$, Rackele Ferreira do Amaral $^{\mathrm{a}}$, Barbara Gomes da Rosa ${ }^{\mathrm{a}, \mathrm{c}}$, Izabella Grimaldi $^{\mathrm{a}}$, Felipe Sceanu Leser ${ }^{\mathrm{a}}$, José Marcos Janeiro ${ }^{\mathrm{a}}$, Lucy Macharia ${ }^{\mathrm{b}}$, Caroline Wanjiru ${ }^{\mathrm{b}}$, Claudia Maria Pereira $^{\mathrm{d}}$, Vivaldo Moura-Neto $^{\mathrm{b}, \mathrm{c}}$, Catarina Freitas $^{\mathrm{c}}$ and Flavia Regina Souza Lima ${ }^{\mathrm{a}}$. $^{*}$

${ }^{\text {a }}$ Glial Cell Biology Lab - Biomedical Sciences Institute, Federal University of Rio de Janeiro, Rio de Janeiro, RJ, Brazil

b Brain's Biomedicine Lab - Paulo Niemeyer's Brain State Institute, Rio de Janeiro, RJ, Brazil

${ }^{c}$ Cellular Morphogenesis Lab - Biomedical Sciences Institute, Federal University of Rio de Janeiro, Rio de Janeiro, RJ, Brazil

${ }^{\mathrm{d}}$ Genetics Laboratory - Faculdade de Odontologia, Universidade do Grande Rio (Unigranrio), Rio de Janeiro, RJ, Brazil

\#Current address: Inserm U970, Paris Cardiovascular Research Center, Paris 75015, France

*Correspondence: flima@ icb.ufrj.br (F.R.S. Lima) and lh_geraldo@icb.ufrj.br (L.H.M. Geraldo)

\section{Abstract}

Glioblastoma (GBM) is the most common and fatal primary malignant brain tumor. Despite advances in the understanding of the biology of gliomas, little has changed in the treatment of these tumors in the past decade. After phase III clinical trials showed no benefit for the use of Bevacizumab in newly diagnosed patients, there is renewed search for new anti-angiogenic drugs, as well as immunotherapeutic approaches, including checkpoint inhibitors, Chimeric Antigen Receptor T cells and intracerebral CpG-oligodeoxynucleotides. The emerging role of infiltrating microglia and macrophages, and of metabolic alterations is also being taken into account in pre-clinical research and drug development. In this review, we discuss progress in the search for new therapeutic strategies, in particular approaches focusing on the tumor microenvironment. 


\section{Keywords: Glioblastoma, Cancer Therapy, Immunotherapy, Microglia, Angiogenesis,}

Cancer Metabolism.

\section{Glioblastoma's heterogeneity and the search for new therapeutics}

GBM is the most common and deadliest primary malignant tumor of the central nervous system (CNS), with an incidence rate of 3.20 cases per 100,000 habitants and a 5-year survival rate of $5.5 \%$ in the United States (US) [1].

According to the World Health Organization (WHO) classification, these tumors are grade IV diffuse astrocytic tumors presenting nuclear atypia, cellular pleomorphism and intense mitotic activity associated with necrosis and microvascular proliferation [2, 3]. This traditional approach to the diagnosis of the CNS tumors was based mostly on histopathological criteria established in the 1920's by the classification system developed by Bailey and Cushing [4]. In accordance with this criteria, tumors should be classified following the histological similarity to the tissues of supposed origin and highlighted by specific microscopic features using lineage specific phenotypic markers [2]. This histological classification and the grading system have been officially used for almost a century because they succeeded in correlating with prognosis and have remained relatively helpful in determining which treatment should be dispensed to the patients, despite some incongruities. However, the great heterogeneity of those tumors often leads to multiple types of diagnosis, reflected by the inter-observer divergences on the final classification [5].

Given these challenges and the increasing knowledge about glioma's biology, in 2016, WHO changed the Central Nervous System (CNS) Tumors Classification to a molecular categorization based on genetic and epigenetic features [3]. Nevertheless, most of the changes were in the diagnosis of low-grade gliomas, now mostly based on genetic alterations rather than morphological status: 
Gliomas bearing the $1 \mathrm{p} / 19 \mathrm{q}$ codeletion should be classified as oligodendrogliomas $[6,7]$. Diffuse astrocytomas bearing the Isocitrate Dehydrogenase (IDH) mutations but lacking the 1p/19q codeletion should be classified merely as astrocytoma. Astrocytoma diagnosis also relies on the presence of two other mutations, at the TP53 gene and ATRX [3]. Both are mutually exclusive to 1p19q codeletion and, if they are present in IDH-mutant gliomas, the diagnosis of oligodendroglioma is immediately excluded [8-10].

The GBMs are the class that has changed the least in this new classification: their diagnosis remains mostly histological but IDH-wild-type tumors correspond to clinically-defined primary or de novo GBM, and those bearing the mutant IDH isoform are designated as secondary GBM, meaning they progressed from low-grade gliomas to GBM [11].

The origin of GBMs is still a matter of debate. Initially, it was suggested that astrocytes, the only cells known to replicate in the adult brain, would suffer oncogenic alterations and give rise to GBM [12]. Other hypothesis suggests that these tumors may derive from precursors, such as Neuronal or oligodendrocyte Progenitor Cells (NPCs or OPCs) [13, 14], but the cellular heterogeneity of GBM makes it extremely hard to precisely identify its cell of origin. More recently, glioma stem cells (GSCs) with self-renewal ability and capability to form tumors in vivo with the same characteristics from the primary tumor were also identified $[15,16]$. Although those are tumorpropagating cells (and not tumor-initiating), their presence has been associated with heterogeneity of these tumors, since they can divide and differentiate asymmetrically and be more resistant to conventional chemotherapy [17]. In GBM we can distinguish between intertumoral and intratumoral heterogeneity. The first refers to the distinct genetic alterations that occur in individual tumors, allowing molecular subgroup classification. On the other hand, intratumoral heterogeneity refers to the diversity within individual tumors. It is related both to the diversity of tumor cellular phenotypes that compose the tumor mass $[18,20-22]$ and to the other cellular entities recruited to the tumor microenvironment, such as microglia/macrophages and endothelial cells [23-25]. 
Currently, standard therapy for newly diagnosed GBM patients consists of: maximal safe surgical resection + Standard or Hypofractionated brain radiotherapy $(\mathrm{RT})+$ concurrent and adjuvant temozolomide $(\mathrm{TMZ})+$ Alternating Electric Field Therapy (or TTFields), even for elderly patients [26-28]. The mechanisms of this treatment are further explained in Box 1. Despite this aggressive treatment, virtually all patients experience tumor recurrence [29-32]. The basis for GBM drug resistance is further explained in Box 2, where we review recent strategies attempting to overcome this issue. Another important fact is that in order for being effective against gliomas, drugs or cell therapies need to effectively cross the BBB. The need to overcome the BBB is a main obstacle that halted the development of numerous treatments. In Box 3 we review some recent and innovative delivery strategies.

Despite all efforts over the last decade to develop improved treatment for GBM patients through the discovery of new chemotherapeutic agents or new approaches, their success is markedly lower than several therapies applied to other cancers, such as breast and gastrointestinal tumors. While there is still a long way to go in the battle against GBM, many scientific advances have been made in recent years leading to new discoveries that can hopefully change the prognostic of GBM patients. They will be revised in the following sections.

\section{Immunotherapy: The next frontier?}

The immune system is an essential component for tumor development and progression, especially in highly heterogeneous tumors as GBM. [33-36]. The immunosuppression is currently recognized as a cancer hallmark present in the Escape phase, where specific cells and molecules, such as M2 macrophages, regulatory T-cells, myeloid-derived suppressor cells and immune checkpoints are present $[37,38]$. The GBMs' microenvironment is characterized by an important immunosuppressive infiltrate [33-36]. Several immunotherapies have already been approved by regulatory agencies in the United States, such as cancer vaccines, immune checkpoint inhibitors, bi-specific 
T-cell engager monoclonal antibodies, oncolytic virus and adoptive cell transfer therapies [39], summarized in Figure 1.

\section{Immune checkpoint inhibitors}

Immune checkpoints are negative immunological regulators. The most studied immune checkpoint receptors are cytotoxic T-lymphocyte-associated antigen 4 (CTLA-4) and programmed cell death protein 1 (PD-1) [40-42]. Over the last years, there was a marked increase in the development of clinical trials based on the blockade of immune checkpoints. A few of them were well succeeded and the Federal Drug Administration (FDA) approved their use for several types of cancer. This is the case of Ipilimumab (a CTLA-4 inhibitor); Pembrolizumab and Nivolumab (PD-1 inhibitors); Atezolizumab, Avelumab and Durvalumab (PD-L1 inhibitors). There are currently nineteen recruiting trials, five active clinical trials of PD-1/PD-L1 in GBM and one completed Phase 2 clinical trial of neoadjuvant Nivolumab. All of these have no published results as yet.

Notwithstanding, a retrospective study showed no Overall Survival (OS) or Progression-free Survival (PFS) benefit in using Nivolumab in patients with recurrent GBM after anti-angiogenic treatment with neutralizing antibodies against VEGF (Bevacizumab) [43]. Nevertheless, initial results from the Checkmate 143 Clinical Trial (NCT02017717) demonstrated the safety of Nivolumab and Iplimumab in GBM patients. In addition, a low response rate to PD-1 inhibitors for patients with High-Grade Gliomas (HGG) was also verified, although in this study a small number of patients clearly benefited from the treatment, having good partial responses and stable disease with increased PFS [44].

Limited $\mathrm{T}$ cell infiltration and activation in the immunosuppressive microenvironment as well as the paucity of validated biomarkers might explain the difficulties encountered in GBM. Strategies to overcome these complications may lay on the combination of therapies, such as immunotherapy with stereotactic radiosurgery or local chemotherapy [45]. 


\section{Intracerebral CpG-ODN: a promise that went wrong?}

Oligodeoxynucleotides containing $\mathrm{CpG}$ motifs (CpG-ODN) derived from bacterial DNA bind to intracellular Toll-like receptor 9 (TLR9) and act as potent activators of innate and adaptive immune systems, triggering a lymphocyte (Th1) response [46]. CpG-ODNs have already been successfully used in diverse experimental models of viral infections, allergies and cancer. Preclinical studies showed that CpG-1826 prolongs survival of mice with gliomas, by inducing tumor cell apoptosis and stimulation of the immune response [47]. Subcutaneous vaccination with tumor cell lysate plus CpG-2006 increased mice survival [48]. The use of multiple low-dose intratumoral CpG-ODN, to avoid toxicity, could lead to tumor eradication and induce long-term remission (longer than 3 months), with an important role of NK cell activation in this process [49]. In addition, radiosensitizing effects that result in inhibition of tumor growth and increased survival have been attributed to $\mathrm{CpG}-28$ and $\mathrm{CpG}-107$ [50-52]. A role in potentiating chemotherapy has also been recently described, using the combination of cyclophosphamide/CpG-1826 and TMZ/CpG-28 [53, 54].

Finally, the delivery mode of CpG-ODN is relevant, since TLR9 is located intracellularly. Both the uptake and immunopotency have been enhanced using carbon nanotubes conjugated with CpG [55]. Multifunctional lipid nanocapsules loaded with paclitaxel and CpG-1826 locally injected via Convention Enhanced Delivery (CED) also increased OS in glioma-bearing mice [56].

There are so far 3 clinical trials using CpG-ODN on GBM patients, which stated that CpG28 is well tolerated at doses up to $20 \mathrm{mg}$ per injection [57], with only modest activity on the 6month PFS [58] and no improvement in OS [59]. Despite the relative success in animal studies, CpG-ODN results in patients have been disappointing. Among conceivable causes of failure, authors point out the delivery method used and the possibly variable TLR9 expression among tumors. Given this data, the improvement in the delivery method of CpG-28 and its association with immune checkpoint inhibitors might lead to more effective outcomes. 


\section{Rindopepimut ${ }^{\circledR}$ : the EGFRvIII mutation vaccine}

Cancer vaccines are another modality of immunotherapy designed to strengthen the patient anti-tumor immune response. Therapeutic vaccination can be done with tumor cell vaccines (autologous and allogenic), dendritic cell vaccines and protein/peptide-based vaccines [60]. It mobilizes various immune effector mechanisms to attack and destroy tumor cells while sparing non-tumor cells [60]. EGFRvIII is a tumor-specific EGFR mutation frequently observed in GBM [61, 62], consisting of an in-frame deletion of $801 \mathrm{bp}$ from the extracellular ligand-binding domain (exons 2-7) that removes amino acids 6-273, inserts a new glycine at the fusion junction [63] and encodes a constitutively active tyrosine kinase associated with tumor aggressiveness and treatment resistance $[64,65]$.

EGFRvIII is an ideal candidate for a peptide-based vaccine due to its tumor-specific nature and high immunogenicity. Rindopepimut ${ }^{\circledR}$, the EGFRvIII-targeting vaccine developed by Celldex Therapeutics, is composed of 14 peptides conjugated to the immunogenic carrier protein keyhole limpet hemocyanin and proved to be efficient in preclinical murine models, but in some cases the treatment failured due to the outgrowth of EGFRvIII-negative cells [66].

Clinical trials of Rindopepimut ${ }^{\circledR}$ in GBM patients (ACTIVATE, ACT II/III and ReACT) had impressive results which led to Breakthrough Therapy Designation by the FDA in February 2015, despite pending results from the final Phase III ACT IV trial in newly diagnosed GBM patients [67-69]. However, the ACT IV phase III trial was terminated earlier for lack of benefit. Final analysis showed no OS benefit and a slight increase in side-effects [70]. In a small group of patients submitted to second surgery during the trial, almost $60 \%$ had lost EGFRvIII expression (57\% in the Rindopepimut ${ }^{\circledR}$ arm and 59\% in the placebo arm), raising the question of the importance of GBM's heterogeneity when choosing an immunotherapeutic target. Given that this was the first phase III trial of Rindopepimut ${ }^{\circledR}$, further studies will be needed to evaluate which subgroups of patients will likely benefit from this vaccine. Moreover, it will be important to establish the role of the highly 
immunosupressive GBM microenvironment in the absence of response to the vaccine and other combination approaches to increase the efficacy of immunotherapy in GBM.

\section{Chimeric Antigen Receptor (CAR)-T adoptive cell therapy}

There is also the adoptive immunotherapy, which consists of artificially activating patient's lymphocytes or dendritic cells to elicit anti-tumor response. Adoptive immunotherapy has gained increasing attention in the last years, as it obviates the multiple steps involved in the stimulation of a primary anti-tumor immune response, while being potentially faster and more effective than immune checkpoint blockade and vaccines. CAR constructs for redirecting $\mathrm{T}$ cells have been the most successful form for redirected adoptive immunotherapy. They consist of an extracellular antigenrecognition domain (usually an antibody single chain variable segment - scFv) linked to the intracellular signaling domain of the $\mathrm{T}$ cell receptor (TCR) and co-stimulatory domains. This allows engineered $\mathrm{T}$ cells to target an antigen independently from MHC exposure, provided that the antigen is a tumor-specific one to avoid off-tumor/on-target effects [71-73].

Since the EGFRvIII mutation is a tumor-specific antigen in GBM patients and is also expressed in treatment resistant GSCs [61, 62], a first-in-human study using peripherally infused EGFRvIII CAR-T cells for recurrent GBM patients was recently reported [76]. CAR-T cells were able to infiltrate the tumor mass, but induced alterations in the tumor microenvironment, such as loss of antigen expression in tumor cells and greatly increased both PD-L1 expression and regulatory FoxP ${ }^{+} \mathrm{T}$ cell infiltration [76].

CAR-T-Cells for other tumor-associated antigens such as EphA2, HER2 and IL-13 Receptor $\alpha 2$ (IL-13R $\alpha 2$ ) are also being tested [76-80]. A Phase I clinical trial using IL13BB $\zeta-C A R-T$ cells (targeting IL-13R $\alpha 2$ ) administered post-surgery inside the resection cavity and intraventricularly in patients with recurrent GBM reported a first-in-human unprecedented transient complete response. Intracavitary doses induced local disease regression and intraventricular doses induced regression of 
distant metastasis (even spinal metastasis), with outstanding improvement in the quality of life (including discontinuation of glucocorticoids and return to normal life activities) [81].

Therefore, the use of CAR-T cells as an immunotherapeutic approach seems promising for GBM, especially when locally administered. Apparently, this modality of treatment is able to somehow overcome the highly immunosuppressive and complex GBM microenvironment, the major barrier to successful immunotherapy. The next years will determine if the use of intracerebral and intraventricular CAR-T cells is indeed safe and feasible and if this strategy induces sustained responses.

\section{Oncolytic virus therapy}

Cancer immunotherapy using oncolytic viruses has gained attention. These viruses possess the ability of infecting and killing tumor cells without causing damage to normal tissues [82]. A potential candidate to this new therapeutic strategy is the rodent $\mathrm{H}-1$ protoparvovirus $(\mathrm{H}-1 \mathrm{PV})$, the smallest among all oncolytic viruses and nonpathogenic for humans [83]. In addition, in gliomabearing immunocompetent models, proinflammatory cytokine release and upregulated CD80 expression were detected in antigen-presenting cells, including microglia [84]. Recently, a phase I GBM trial showed that patients treated with parvovirus increased tumor infiltrated lymphocytes and extended median survival [85].

Other oncolytic viruses demonstrated the ability to induce an effective immune response against tumor invasion. In a mouse GBM model, the intratumoral administration of an oncolytic adenovirus stimulated IFN- $\gamma$-producing CD8+ $\mathrm{T}$ cells and decreased tumor-infiltrating Treg cell numbers [86]. Moreover, a recombinant polio/rhinovirus chimera also demonstrated immunotherapeutical potential in vitro in high-grade GBM [87].

\section{Targeting angiogenesis: Bevacizumab and beyond}


Angiogenesis is a complex mechanism of tumor growth in brain tumors due to intricate interactions between endothelial cells and other entities from the GBM microenvironment (particularly tumor cells and macrophages/microglia). GBM vascularization occurs through several alternative mechanisms besides sprouting angiogenesis, such as vascular co-option, vascular mimicry, GSC endothelial differentiation, and vasculogenesis from Bone-Marrow (BM)-derived circulating endothelial precursor cells (CEPC), which are all regulated by complex interactions with the tumor microenvironment $[88,89]$. In the hypoxic tumor areas, Vascular Endothelial Growth Factor A (VEGF-A) is upregulated in GBM cells and in infiltrating macrophages, resulting in abnormal microvascular stimulation leading to aberrant highly proliferative, tortuous and leaky vasculature, which further contributes to tumor hypoxia and compromises efficient drug delivery [88-91].

Bevacizumab, an IgG1 monoclonal antibody which neutralizes the effect of VEGF-A, has an inhibitory effect on tumor neovascularization processes. However, Bevacizumab would not inhibit vascular mimicry (when tumor cells acquire genetic and/or phenotypic features of endothelial cells) or the blood supply from pre-existing vasculature [92, 93]. Indeed, histopathological analysis showed that Bevacizumab therapy induces apparent normalization of the vascular structure, decreases microvessel density and improves tumor oxygenation [94].

In 2009, the FDA approved Bevacizumab in monotherapy or combined with chemotherapy for second line treatment of recurrent GBM [29, 30]. Nevertheless, for newly diagnosed GBM patients, adding Bevacizumab to the conventional treatment did not improve OS and was associated with increased side effects. When Bevacizumab was used as first line therapy, patients experienced increased symptom burden, worse quality of life and a more frequent and early decline in neurological function [95]. Given this data, Bevacizumab remains restricted for refractory patients, where it increases PFS, has a corticosteroid sparing effect and improves quality of life, although patients tend to relapse during treatment $[96,97]$.

GBM recurrence after VEGF blockade is often associated with a phenotypic alteration due to glial-mesenchymal transition of tumor cells [98], apparently triggered by MET (a receptor tyro- 
sine kinase of hepatocyte growth factor) phosphorylation. Impairment of VEGF function restores and increases MET activity in a hypoxia-independent manner, resulting in tumor cell invasion and mesenchymal transition through a MET/VEGFR2 complex [99]. Tumor endothelial cells acquire the mesenchymal gene profile without loss of endothelial cell functions, which is defined as endothelial mesenchymal transition (Endo-MT), resulting in enhanced cell proliferation and migration, as well as vessel permeability [100]. These mechanisms are triggered by an axis involving c-MET, ETS-induced 1 and metalloproteinase 14 (MMP14), which also controls VE-cadherin degradation and vascular abnormality. Pharmacological inhibition of c-MET induced vessel normalization in patient tumor-derived endothelial cells. In addition, specific knockout of MET in endothelial cells presented normalized blood vessels, reduced intratumoral hypoxia, which suppressed tumor growth and prolonged survival in GBM-bearing mice after TMZ treatment [100].

Although the anti-angiogenic therapy improves tumor oxygenation, hypoxia persists in the process of tumor resistance to treatment [94]. The hypoxic microenvironment re-induces HIF-1 $\alpha$, resulting in stromal cell-derived factor 1 (SDF-1) and VEGF activation and leading to the migration of pro-angiogenic monocytic cells from the bone marrow, endothelial cells and pericyte progenitors [101]. Another molecule that stimulates angiogenesis in tumor microenvironment by enhancing the VEGF pathways is the placental growth factor (PIGF) [102]. [103] However, no significant clinical activity was observed when the anti-PIGF monoclonal antibody was tested in phase I for patients with recurrent GBM [104].

Even though antiangiogenic therapy has failed to stop GBM progression, the most common strategy to treat recurrent GBM is continuation of Bevacizumab with the addition of a cytotoxic drug [105]. This strategy is still used because lower doses of antiangiogenic therapy normalize tumor microvasculature, which may improve drug delivery and reduce hypoxia [106]. Among the available cytotoxic adjuvants, Lomustine may still have a role in the GBM recurrent setting, since nitrosoureas can easily cross the blood brain barrier [107]. Nevertheless, this effect of chemotherapy is transient and restricted to this "window of normalization" (the time frame where the vascula- 
ture is normalized after anti-angiogenic treatment), that is dependent on both treatment dose and duration [108].

An alternative to treat recurrent GBM is the administration of inhibitors of the angiotensin system (ASI) (prescribed as anti-hypertensive drugs) concomitant with cytotoxic-Bevacizumab therapy [109]. These inhibitors block both the angiotensin II receptor I (AT1) signaling and the angiotensin converting enzyme (ACE). The therapy is associated with improved survival in GBM patients receiving standard-of-care therapy [110]. AT1 inhibition with ASI was previously shown to reduce tumor VEGF levels in preclinical glioma models [111]. ASI treatment may also enhance vascular perfusion and reduce edema in the tumor and peritumor brain, improving drug delivery to the tumor, for a fraction of the cost of standard care treatments and with minimal associated risks $[108,112]$.

Other anti-angiogenic agents could be used as an alternative to the Bevacizumab therapy, such as VEGF receptor tyrosine kinase inhibitors (vatalanib, sorafenib, cediranib, sunitinib and pazopanib), PDGF receptor tyrosine kinases inhibitors (dasatinib, imatinib and tandutinib), protein kinase C inhibitors (enzastaurin), matrix metalloproteinases inhibitors (prinomastat) and proteasome inhibitors (bortezomib) [113-115]. Unfortunately, though many phase I and II studies have been performed, none of these agents has demonstrated to be effective enough to justify a phase III clinical trials [116], as tumors eventually overcome these treatments by the same mechanisms of Bevacizumab resistance.

Tumor cells can evade angiogenic inhibitors through modulation of collateral pathways or co-option of existing normal vasculature to maintain blood perfusion [117]. To overcome the limitations of established antiangiogenic drug therapies, gene therapy was used to target newly formed blood vessels. In a murine model, VB-111, a non-replicating adenovirus 5 carrying a proapoptotic human Fas-chimera transgene (Fas and human TNF receptor 1), was able to specifically act in the expression of the Fas-chimera transgene in angiogenic blood vessels triggering apoptosis of these vessels [118]. 
Given all the data available, the importance of Bevacizumab cannot be put aside. But the lack of a long-lasting response and the increasing understanding of the mechanisms regulating angiogenesis in the GBM highlight the urge to develop and test new anti-angiogenic drugs that would have a longer window of normalization, or would involve alternative pathways that could be responsible for the resistance to VEGF targeting.

\section{Glioma-Associated Macrophages/Microglia (GAMs) as key players in GBM microen- vironment}

Microglia are the resident immune cells on the brain parenchyma and are involved in many cell events in healthy and diseased CNS comprising chemotaxis, phagocytosis, antigen presentation and cytokine production. Although it has been described that tumor-infiltrating microglia and macrophages compose up to $30 \%$ of the tumor mass, those cells do not appear to have antitumor activity. Actually, glioma-associated microglia/macrophages (GAMs) produce pro-tumor factors and contribute to GBM progression [24, 119], view Figure 2.

Histopathological analysis of glioma specimens demonstrated that GBM contains a higher number of infiltrating macrophages compared to lower grade astrocytomas [120]. In the past few years, the activation status of GAMs present in the tumor microenvironment has been considered of prognostic value [121].

Similar to regular macrophages, microglia can acquire an M1 phenotype, that is involved in effective immune response against tumor progression and is characterized by the ability to release pro-inflammatory mediators such as TNF, IL-1, IL-6, CCL2, nitric oxide (NO) and reactive oxygen species (ROS) [122]. However, tumor infiltrating microglia and macrophages mostly also display an M2 phenotype, which possibly contributes to the immunossupressive microenvironment of GBM. These alternatively activated M2 macrophages produce anti-inflammatory and immune suppressive factors, including arginase-1 and CD36, as well as up-regulate the cell surface markers CD204, CD206 and CD163 and anti-inflammatory cytokines, such as IL-10 [123]. In the healthy 
brain, resident microglia (also referred to as M0) are considered to possess an attenuated M2 phenotype and contribute to the maintenance of a healthy environment for neuronal function [124]. On the other hand, GAMs were found to express some M1 but mostly M2 markers in human GBM samples [125].

STAT 3 signaling, which is constitutively expressed in GBM [126], is also up-regulated in GAMs [127-129]. The activation of this pathway inhibits M1 macrophage activation, reduces expression of co-stimulatory molecules necessary for antigen-presentation and upregulates immunomodulatory cytokines IL-6 and IL-10, while down-regulates pro-inflammatory cytokines (IL-2, IL4, IL-12 and IL-15) [130, 131]. Therefore, STAT 3 inhibition using siRNAs reverses the cytokine profile, leading to GAM activation and inhibition of tumor growth in vivo [128]. STAT 3 pharmacological inhibitors penetrate the CNS and have anti-proliferative and pro-apoptotic effect on GBM cells $[131,132]$. These inhibitors are capable of reversing the immune tolerant microenvironment by activating microglial cells, increasing lymphocyte-stimulating cytokies (IL-2, IL-4, IL-12, IL-15 and CXCL-10) and upregulating co-stimulatory molecules (CD80 and CD-86), then inducing a TH1-response $[132,133]$. There are ongoing phase I and II clinical trials with inhibitors of STAT 3 and its most relevant activator, IL-6, for several malignancies such as head and neck cancer, multiple myeloma and prostate cancer [134]. These trials might also be translated into a new therapeutical option for malignant gliomas.

It has been recently demonstrated that hypoxia increased microglia and macrophage recruitment by chemotaxis as well as the tumor expression of POSTN, a cell-secreted adhesion protein that is capable of promoting tumor progression[135]. However, macrophage motility was attenuated by hypoxia, which corroborates with the idea that GAMs are primarily attracted to hypoxic areas and after they remain imprisoned in these areas [135]. In addition, POSTN expression was enhanced through TGF- $\beta$ (a hypoxia-inducible growth factor) treatment, resulting in increased recruitment of GAMs to HIF-1 $\alpha$-positive regions [136]. In addition, hypoxia promotes M2polarization of GAMs and HIF inhibition partially reversed the effects of hypoxia in GAM recruit- 
ment and M2 polarization [136]. These findings suggest a connection between the hypoxic environment with GAM enrichment and M2 polarization, key factors in tumor progression that could be targeted to reduce glioma growth and invasiveness. Finally, a recent work showed that the activity of GBM's iNOS was capable of inhibiting microglial caspase-3, which was a crucial step in the transformation of microglial cells in their tumor-promoting phenotype in vitro and in vivo [137].

Other important pathway in the crosstalk GAM-GBM is the colony stimulating factor 1 (CSF-1), also known as macrophage colony-stimulating factor (M-CSF). CSF-1 is a secreted cytokine that binds to CSF-1R in hematopoietic cells and macrophages stimulating survival, differentiation and proliferation, which have been described to be important in GBM invasion [138, 139]. Furthermore, CSF-1 inhibition can block GBM progression by altering macrophage M2 polarization and increasing survival in preclinical models [121]. In preclinical models, tumors treated with antiCSF-1 recur in over $50 \%$ of mice. CSF-1 inhibition resistance is mediated by the tumor microenvironment, through macrophage production of insulin-like growth factor-1 (IGF-1) and activation of IGF-1 receptor (IGF-1R) in GBM cells. Blockade of IGF-1R or PI3K pathway further prolonged animal survival [140]. Finally, an orally administered CSF-1R inhibitor (PLX3397) is being tested in GBM patients and first results from phase II clinical trials showed that treatment was well tolerated and crossed the blood-brain-barrier but showed no efficacy [141].

The CX3CR1-I249 allele, a recently discovered prognostic marker, is a polymorphism in a chemokine receptor gene associated with cell migration. This variant is associated with prolonged mean OS of GBM patients (23.5 vs 14.1 months) and with reduced tumor infiltration by GAMs [142]. For the first time, a GAM marker has been characterized as an independent, favorable prognostic factor and might be useful in predicting survival in GBM patients.

Another controversy in the literature lies on the differences between microglia and bonemarrow derived macrophages in GBM development. Recent studies using transgenic mice and intra-vital two-photon microscopy gave some insight to this debate: microglial cells and bone-marrow derived macrophages have different transcriptional networks [143] and different spatio-temporal 
recruitment, morphology and activation dynamics [144]. Also, recent evidence is pointing to bonemarrow derived macrophages as important players in tumor angiogenesis. Molecules produced by endothelial cells, such as IL-6, could be relevant for attracting and polarizing these cells towards the M2-tumor promoting phenotype through activation of HIF-2 $\alpha$ [145]. Once in the perivascular compartment, those cells would be an important source of VEGFa for endothelial cells [91]. Recent observation with intra-vital two photon microscopy in a syngeneic mouse glioma model showed that in early stage tumors there was accumulation of M1-polarized macrophages in the tumor core. While in later stage tumors, those cells accumulated in the perivascular region and were predominantly M2-polarized, VEGFa-secreting [91]. Furthermore, this macrophage-derived VEGFa was responsible for blood vessel dysmorphia observed in GBM. Selective ablation of VEGFa in macrophages could reverse this effect and improve chemotherapy deliver and mice survival [91].

All these findings point to the importance of further studies about the influence of the tumor microenvironment on the response of GAMs and the crosstalk between these and tumor cells, as this can be a promising target for cellular and immunotherapy in GBM.

\section{Targeting Reprogrammed Metabolism: IDH1/2 and beyond.}

In order to develop a highly proliferative phenotype, transformed cells experience a metabolic reprogramming, which provides them with required substrates to sustain intense cell division [147]. For many years, glucose was considered the primary energy source for many cancer types, including gliomas, in a process known as the 'Warburg effect' ('aerobic glycolysis') [148]. This effect consists of a shift of the metabolic flux from Oxidative Phosphorylation to fermentation under normal oxygen concentrations [149]. Despite being less efficient, this process confers unique advantages to malignant cells, such as quicker energy gain; acidification of the extracellular microenvironment aiming to facilitating neoplastic invasion; and increased concentrations of glycolytic metabolites and $\mathrm{NADH}$, which will be used to generate aminoacids and fatty acids, ultimately promoting cellular proliferation $[149,150]$. 
To enhance the glycolytic flux, GBM must undergo modifications in protein expression and activity. An elevated glucose transport is guaranteed by increasing the expression of glucose transporters (GLUT1, GLUT3). Elevated glycolytic activity is acquired by expressing unique isoforms of rate-limiting glycolytic enzymes, such as Hexokinase-2, PFKFB3 and PFKFB4, PDK1 and PKM2 [151-153]. Hexokinase-2, which catalyzes the formation of glucose to glucose-6-phosphate (G6P), is not commonly expressed by benign brain tissue and its expression has been correlated with lower GBM patient OS [152]. Knockdown of Hexokinase-2 reduces tumor vasculature and tumor growth in orthotopic xenograft models of GBM, and sensitizes malignant cells to TMZ and $\mathrm{RT}$ in vitro and in vivo [154]. Likewise, PKM2, which catalysis the irreversible phosphorylation of Phosphoenolpyruvate (PEP), is overexpressed in malignant gliomas when compared to normal brain cells [155] or lower-grade gliomas [156]. PFKFBs (PFKFB1, 2, 3 and 4) indirectly regulate the activity of PFK1 by producing and degrading F26BP, a known PFK1's potent activator. Whenever PFK1 is inactive, the addition of one phosphate group to fructose1-phosphate does not occur and the glycolytic flux is reduced [157]. Recently, it was demonstrated that TGF $\beta-1$ increased the glycolytic flux by inducing PFKFB3 expression in GBM [158]. RNAi screening identified PKM2 and PFKFB4 as survival-related kinases in GSC and high PFKFB4 expression was associated with lower survival in GBM patients [159].

Aberrant expression and activity of glycolytic "players" can be therapeutically explored to improve the current therapy of gliomas. Therefore, a number of small-molecule inhibitors has been developed and some of them are currently in phase I/II clinical trial [160]. Lonidamine is a Hexokinase-2 inhibitor, which leads to cellular apoptosis [161] but did not show effective results in phase II trials [162]. Dichloroacetate, a PDK1 inhibitor that increases ROS and mitochondria-dependent apoptosis in vivo [163], is currently under phase II trial for GBM (NCT00540176).

As mentioned in Box 1, gliomas have point mutations in the Isocitrate Dehydrogenase 1 and 2 genes (IDH1-R132H and IDH2-R172K, respectively) [164]. The wild-type enzyme is responsible for converting isocitrate into $\alpha$-ketoglutarate $(\alpha-K G)$, the substrate used for the formation of 
succinyl-CoA in the Tricarboxylic Acid (TCA) cycle. It has been observed that mutated IDH1$\mathrm{R} 132 \mathrm{H}$ acquires a unique capability of transforming $\alpha$-KG into 2-hydroxyglutarate (2HG) [165]. The accumulation of $2 \mathrm{HG}$ by cells bearing the mutated IDH leads to several consequences, including impairment of epigenetic-related enzymes (mainly DNA demethylases), causing a vast rearrangement of the DNA methylation profile $[166,167]$. Considering the importance of IDH/2-HG in the tumorigenesis of gliomas, several research groups developed strategies in order to counteract their activity within transformed cells [168-170]. Some of the studied compounds are already under phase I clinical trial studies, such as: AG-120 (NCT02073994), AG-881 (NCT02481154) and BAY1436032 (NCT02746081).

Although glutamine rather than glucose can be used as an energy source by GBM [171, 172], it is unknown if ketone bodies can be used as well. GBM cells are sensitive to glucose depletion and undergo high oxidative stress in the absence of this substrate, while normal astrocytes maintain their metabolic balance [173]. A number of studies observed higher glycemia as a predictor of lower OS in GBM patients $[174,175]$. It has been proposed that a dietetic intervention based on low glycemic nutrients (Ketogenic Metabolic Therapy; KMT), might hold GBM progression and increase patient OS [176]. A recent systematic study reviewing current trials testing KMT in gliomas suggested that KMT is safe, feasible and stand as a potential strategy for combined therapy with current treatments in malignant gliomas [177].

Research on cancer cell metabolism has grown exponentially in the past few years and advances in laboratory techniques allowed the development of more reliable in vitro and in vivo models [178]. Several potential new metabolic targets are discovered every year and a great number of them are currently under clinical trial and will hopefully, in a near future, improve the care of GBM patients.

\section{Small non-coding RNAs}


Non-coding RNAs are a family of RNAs that are not transcribed into proteins and include miRNAs, siRNAs and lncRNAs [179]. They participate in diverse biological processes, including control of gene expression, during development and both physiological and pathological process [179]. miRNAs are the most extensively ncRNAs studied and correspond to a class of small ( 22 nucleotides in length), endogenous, evolutionarily conserved RNAs, that regulate gene expression in a post-transcriptional manner by binding to the target mRNAs, thereby repressing their translation or inducing their degradation [180]. Also, miRNAs have become particularly appealing as biomarkers due to their availability and stability in a variety of biological samples (such as blood and saliva) allowing their use as a noninvasive tool for cancer detection and follow-up [181, 182] and as promising therapeutic targets in gliomas $[183,184]$.

In GBM, oncogenic actions of miRNAs such as miR-10b [185, 186], miR-21 [187] and miR-26a [188] have been correlated with inhibition of key cell cycle inhibitors and pro-apoptotic genes [185] and a reduced median OS in GBM patients [188]. Tumor suppressor miRNAs such as miR-7 [189], miR-34a [190], miR-218 [191] and miR-873 [192] have been described as downregulated in gliomas when compared to adjacent or normal brain tissue.

miRNAs also participate in several signaling programs of GSCs [193, 194]: miR-125b seems to be involved in the development, migration and replication of GSCs [195]. Ectopic overexpression of microRNA-34a led to inhibition of proliferation in GSCs by targeting Rictor, a component of mTORC2 complex, promoting suppression of AKT and inhibition of WNT/ $\beta$-catenin signaling pathway [190]. miR-9/9* was also shown to inhibit GSCs differentiation, maintaining their stemness by targeting CAMTA1, a tumor suppressor gene correlated with survival [196]. miR143 is another miRNA related with GSCs which participate in glucose metabolism. Overexpression of miR-143 has been shown to inhibit glycolysis, promoting differentiation and decreasing the tumorigenic capacity of GSCs in vivo, therefore highlighting the important role of this miRNA as a tumor suppressor [197]. 
Some studies suggest that miRNA expression profiles can be used to distinguish glioma grades and GBM subgroups [198, 199]. Recently, a large scale assessment using 848 human microRNAs, observed that a set of 13 microRNAs (miR-1296, miR-21, miR-155, miR-21*, miR-451, miR-223, miR-210, miR-493, miR-16, miR-22, miR-629*, miR-105, and miR-219-2-3p) had a significant different expression patterns, distinguishing WHO grade II glioma from grade III + IV tumors. A higher expression of miR-21 and miR-210 was also associated with worse OS in GBM patients, suggesting a direct involvement of these miRNAs in glioma development and progression [200]. Visani and co-workers also demonstrated deregulation of three microRNAs (miR-34a, miR-101 and miR-10b) between low and high grade gliomas, with higher expression levels of miR-10b being associated with malignancy, representing a promising target for therapy [201].

Furthermore, siRNAs are promising therapeutic tools due to the possibility of being chemically synthesized and artificially introduced into the cytoplasm of cells to inhibit targeted protein synthesis [202].

Some targets are showing promising results in the pre-clinical setting, such as intratumoral treatment with siRNA targeting HIF-1 $\alpha$, which could reduce $79 \%$ of the tumor growth after 50 days of treatment in orthotropic murine models, reducing simultaneously HIF- $1 \alpha$ main transcriptional targets, like VEGF, GLUT-1, c-MET and CA-IX. [203]. Cell penetrating peptide-mediated siRNA inhibition of Hsp90 in combination with 17-allylamino-17-demethoxygeldanamycin (17-AAG), an HSP90 inhibitor, reduced GBM tumor growth via akt downregulation [204]. siRNAs anti-Galectin1 and anti-EGFR were both carried by lipid nanocapsules and administrated intratumorally together with TMZ via CED in an orthotopic GBM mouse model, leading to significantly higher survival [205].

Despite the promising scenario of ncRNAs therapeutic use, several limitations, including an inadequate delivery of a siRNA to the brain tumor tissue and the stability of these molecules in systemic circulation, have been discussed for RNAi based-therapy in GBMs [202, 206]. Particularly, 
more attention should be paid to unpredictable effects, since one miRNA can target multiple mRNAs, and a single mRNA can also be targeted by multiple miRNAs [202].

\section{Concluding Remarks}

Numerous recent studies increased the understanding of the cellular and molecular events involved in CNS oncogenesis. This led to the major changes in tumor classification made by the WHO in 2016 and to the understanding that tumors with different origins and molecular alterations must not be treated in the same way but instead through personalized therapy. Many promising basic and translational investigations have showed no benefit when translated to patient settings, forcing the scientific community to further concerning and engagement towards unveiling the intricate pathways involved in GBM establishment and progression, but many questions remain to be addressed (see Outstanding Questions).

Due to the heterogeneity of GBM, the complex interactions between tumor and parenchymal entities are gaining exponential importance. Unlike many other solid tumors, the GBM microenvironment lacks lymphocyte infiltration while it is abundant in macrophages, which can be pointed as one of the causes of the poor outcome of immunotherapy in these tumors. In this sense, a great deal of the research carried on in the field currently aims at better understanding the role of innate immune cells in the GBM microenvironment, particularly in how those cells are polarized to M2 phenotype after their recruitment and how they interact with other cells from the tumor microenvironment, specially endothelial cells and the few infiltrating lymphocytes. New therapies targeting the myeloid compartment of the tumor microenvironment can hopefully be a viable and effective alternative for treating these tumors as a new line of immunotherapy. Also, targeting the myeloid compartment of the tumor microenvironment can improve the success of immunotherapy with checkpoint inhibitors, by increasing possibility of $\mathrm{T}$ cell recruitment and changing the immunossupressive tumor microenvironment. 
Also, research in tumor angiogenesis is now evolving from the focus on the VEFGA/VEGFR2 axis; as targeting this pathway was proven insufficient to properly solve blood vessel dysmorphia and dysfunction in the tumor microenvironment. The search for alternative pro and anti-angiogenic pathways is being further pursued and may also lead to a better understanding on the role of the interaction between macrophages/microglia and endothelial cells, a topic already explored in developmental angiogenesis studies but still largely neglected in the context of GBM and other malignancies.

Finally, recent advances in metabolomics are allowing researchers to see beyond the Warburg Effect in the cancer field. Better understanding the differential expression of distinct enzyme isoforms and the metabolic deviations that occur in tumor cells is helping identifying metabolites with different cellular activities. It can also show what are the crucial steps in the metabolic cascade that should be targeted for inhibiting tumor growth.

\section{Acknowledgments}

This study was supported by the Brazilian Funding agencies Conselho Nacional de Desenvolvimento Científico e Tecnológico (CNPq), Coordenação de Aperfeiçoamento de Pessoal de Nível Superior (CAPES), Fundação de Amparo à Pesquisa do Rio de Janeiro (FAPERJ), Instituto Nacional de Neurociência Translacional (INNT) and Programa de Pós-Graduação em Ciências Morfológicas (PCM), UFRJ. We also thank Dr. Thomas Mathivet for the critical reading of this manuscript.

\section{Text Boxes:}

\section{Box 01 - Molecular Basis of Current Standard Therapy}

TMZ is an orally available DNA alkylating agent [207] with radiosensitizing effect [208]. The principal mechanism of action of TMZ is adding methyl groups to N7 and O6 positions of gua- 
nine and N3 of adenine in DNA [209]. In addition, it has a cytotoxic effect against malignant cells, resulting in DNA fragmentation and disruption of DNA replication, thus leading to growth suppression that causes apoptotic cell death [210]. However, this DNA methylation can be mended by the O6-methylguanine-DNA methyltransferase (MGMT) repair enzyme, thereby suppressing cytotoxicity [211]. Several patients with GBM show resistance to TMZ due to the expression and methylation status of the MGMT promoter [212]. Patients who have tumors with the MGMT promoter methylation usually experienced improved survival when they were treated with alkylating agents like TMZ, making MGMT methylation a critical biomarker for predicting sensitivity to TMZ therapy [213].

Alternating electric fields therapy (TTFields) is a new modality of anticancer treatment, developed by Dr. Yorum Palti [214-216]. It, consists of electric fields of frequencies between 100 and $300 \mathrm{kHz}$, capable of inducing violent membrane blebbing, disruption of microtubule spindle elements and mitotic chromosomal order [214]. In vitro and in vivo models showed that TTFields can inhibit GBM cell migration, invasion and angiogenesis, making it a promising new therapeutic strategy, with multiple biological effects [217, 218]. Of great importance is the fact that the most common adverse event associated with the device is scalp irritation and contact dermatitis due to hydrogel-induced irritation, which could be overcame with topical corticosteroids and slight shifts of the arrays [219].

Recent clinical trials proved this therapy to be effective both in relapsed patients and in the first line setting [27, 215]. Furthermore, continuation of TFF therapy after first recurrence, associated with a second-line chemotherapy is also associated with an increase in OS (11.8 vs 9.2 months) [220]. Despite being the first new treatment approved for GBM patients in the first-line setting after the Stupp Regimen, the use of TTFields is still under great debate, due to the prohibitive cost of the device, approximately 185,476 Euros per patient per year [221].

\section{Box 02 - The old problem of drug resistance in GBM}


One major cause of treatment failure in high grade gliomas continues to be tumor cell resistance to chemotherapeutic agents (multidrug resistance - MDR) [222]. A multifactorial phenomenon which involves several mechanisms that result in evasion of drug-induced cellular damage [223]. The mechanisms of MDR involve deregulation of genes controlling apoptosis, enhanced intracellular drug detoxification, upregulation of DNA repair related genes, activation or overexpression of drug export proteins that reduce levels of drug accumulation in the cell [224].

One category of drivers of the MDR phenotype are the drug efflux proteins that belong to a super family class of molecular pumps known as ATP-binding cassettes transporters (ABC- transporters). Their function is mainly to protect cells from endogenous toxins, xenobiotics and in the transport of many cellular products [225].

High expression of ABC transporters has been shown in the CNS and brain tumors [226]. The transporter P-glycoprotein (P-gp/ ABCB1) is highly expressed on endothelial cells of the blood-brain barrier, while the transporter multidrug resistant-like protein (MRP-1 /ABCC1) and breast cancer associated protein (BRCP/ABCG2) are expressed on lower levels in astrocytes [227]. High levels of expression of multidrug transporters are often observed in drug-naive tumors, even when the tissue of origin exhibits little or no expression of the corresponding gene [228]. This high intrinsic gene expression is likely regulated by signaling pathway components that are involved in malignant transformation. Indeed, recent studies have shown that P-gp over expression in the TMZ resistant GBM cells was caused by an increase in EGFR signaling [229]. Competitive binding assays and specific P-gp inhibition experiments have demonstrated that TMZ is a substrate for P-gp and not MRP-1 [230]. Clinical data suggest that polymorphisms of P-gp have an important role in patient response efficiency to TMZ: tissue samples from patients who exhibited TMZ resistance and tumor recurrence showed increased levels of P-gp expression [231].

The complete profile of substrates for all the ABC transporters associated with MDR is not yet known and despite conflicting information on clinical significance of targeting $\mathrm{ABC}$ transporters, more studies are still underway to fully understand the molecular pathways leading to 
their overexpression in tumors. This data would be important in elucidating the mechanisms underlying the resistance of GBM to chemotherapeutic drugs and subsequently establish the basis for selecting better chemotherapeutic agents and re-sensitizing GBM cells to chemotherapy [232]

\section{Box 03 - Overcoming the Blood Brain Barrier: New Delivery strategies for brain tumors.}

\section{A) Nanoparticles and Micelles:}

Nanoparticles are materials from one to a hundred nanometers, large surface-to-volume rate and capable of crossing the BBB to some extent [233]. Considering the case of brain tumors, where some promising drugs are abandoned during pre-clinical development due to their inability to reach cytotoxic concentrations in the CNS or inability to cross the $\mathrm{BBB}$, these molecules can be of great value [233]. Apart from the nanoparticles, micelles (spherical-shaped aggregates of surfactant molecules, mainly lipids, dispersed in a liquid colloid) are also being loaded with drugs and used to increase $\mathrm{BBB}$ penetration.

To this purpose, some studies have focused in improving TMZ delivery and reducing sideeffects in GBM in vivo models using different strategies (immunoliposomenanocomplex; biotinfunctionalized chitosan core covalently conjugated with TMZ) [234, 235]. Micelles carrying flavonoids or traditional chemotherapy (doxorubicin and paclitaxel) showed in vitro and in vivo antiGBM activity [236, 237].

Therefore, preclinical studies point nanoparticles and micelles as possible powerful tools to improve drug delivery in GBM, with further studies needed to prove their feasibility.

\section{B) Local infusion and Blood-Brain-Barrier Disruption:}

Convection-Enhanced Delivery (CED) is a local delivery technique for facilitating drug diffusion to brain tumors. It allows a direct delivery of high doses by stereotactically inserting catheters into tumor mass or surgical resection cavity [238]. Those catheters are then connected to an infusion pump, which guarantees a positive pressure gradient, to improve drug distribution independently of the agent's molecular weight, allowing infusion of higher volumes [239, 240]. 
Between 1997 and 2010, there were 14 clinical trials involving conjugated toxins, viruses, liposomes and conventional chemotherapies unable to penetrate BBB. All these studies included a limited number of patients and the results were mostly inconclusive [241]. Despite limited success of those clinical trials, the use of CED for GBM treatment remains relatively promising. Notwithstanding, the choice of agent, cannula design and placement and the cost of the procedure constitute some of the challenges that still need to be overcome.

Furthermore, recent studies have focused on local and temporary BBB disruption, aiming to increase drug concentration in the brain parenchyma and inside the tumor mass. In one of these studies, the MRI-guided laser interstitial thermal therapy was able to induce disruption of peritumoral BBB, mainly between the first $1-3$ weeks and decreasing until the 6th week in 14 patients with recurrent GBM., which could open a time window for optimal tumor treatment [242].

\section{Figure Legends:}

Figure 01. New therapeutic strategies for GBM treatment. (A and B) Current immunotherapy strategies being tested pre-clinically and in Clinical Trials, including adoptive cell-transfer (CAR-T cell therapy) and immune-checkpoint blockade. (C) Vaccination strategy targeting EGFRvIII mutation (Rindopepimur@) and anti-angiogenic strategies (D).

Figure 02. Glioma-associated microglia and macrophages infiltrate GBM mass and interact with tumor cells. Confocal microscopy of GBM-injected Swiss mice brain stained for microglia and macrophages with anti-Iba1 (Garcia and Geraldo, unpublished) showing ramified morphology typical of non-activated or "resting" microglial cells, engaged in immuno-surveillance of the CNS (A); and ameboid activated glioblastoma-infiltrating macrophages and microglia (B). (C-E) In several injury settings, infiltrating macrophages and microglial cells can acquire different activation phenotypes: classical M1 phenotype was described in infectious diseases as a response to bacterial 
LPS for example; alternately, activated M2 cells were described in the context of tissue repair. Currently it is known that microglial cells can shift phenotypes during a single pathological process. Iin the GBM tumor mass, for instance, one can identify microglia and macrophages secreting both M1 (IL-2, IL-4, IL-12 and IL-15) and/or with M2 (IL-10 and TGF $\beta$ ) cytokines and other growth factors important for tumor growth, such as VEGF.

\section{References:}

1 Ostrom, Q.T., et al. (2016) CBTRUS Statistical Report: Primary Brain and Other Central Nervous System Tumors Diagnosed in the United States in 2009-2013. Neuro Oncology 18, v1-v75 2 Louis DN, O.H., Wiestler OD, Cavenee WK, Burger PC, Jouvet A, Scheithauer BW, Kleihues P (2007) The 2007 WHO classification of tumours of the central nervous system. Acta Neuropathol 114,13

3 Louis, D.N., et al. (2016) The 2016 World Health Organization Classification of Tumors of the Central Nervous System: a summary. pp. 803-820

4 Bailey, P. and Cushing, H. (1926) A classification of tumors of the glioma group on a histogenetic basis with a correlated study of prognosis. JB Lippincott

5 van den Bent, M.J. (2010) Interobserver variation of the histopathological diagnosis in clinical trials on glioma: a clinician's perspective. Acta Neuropathol 120, 297-304

6 Brat, D.J., et al. (2015) Comprehensive, Integrative Genomic Analysis of Diffuse Lower-Grade Gliomas. N Engl J Med 372, 2481-2498

7 Eckel-Passow, J.E., et al. (2015) Glioma Groups Based on 1p/19q, IDH, and TERT Promoter Mutations in Tumors. N Engl J Med 372, 2499-2508

8 Abedalthagafi, M., et al. (2013) The alternative lengthening of telomere phenotype is significantly associated with loss of ATRX expression in high-grade pediatric and adult astrocytomas: a multiinstitutional study of 214 astrocytomas. Modern Pathology 26, 1425-1432

9 Nguyen, D.N., et al. (2013) Molecular and morphologic correlates of the alternative lengthening of telomeres phenotype in high-grade astrocytomas. Brain Pathology 23, 237-243

10 Reuss, D.E., et al. (2015) ATRX and IDH1-R132H immunohistochemistry with subsequent copy number analysis and IDH sequencing as a basis for an "integrated" diagnostic approach for adult astrocytoma, oligodendroglioma and glioblastoma. Acta Neuropathologica 129, 133-146 11 Ohgaki, H. and Kleihues, P. (2013) The definition of primary and secondary glioblastoma. pp. 764-772

12 Visvader, J.E. (2011) Cells of origin in cancer. Nature 469, 314-322

$13 \mathrm{Liu}$, C., et al. (2011) Mosaic analysis with double markers reveals tumor cell of origin in glioma. Cell 146, 209-221

14 Alcantara Llaguno, S.R. and Parada, L.F. (2016) Cell of origin of glioma: biological and clinical implications. British Journal of Cancer 115, 1445-1450

15 Singh, S.K., et al. (2004) Identification of human brain tumour initiating cells. Nature 432, 396401

16 Yuan, X., et al. (2004) Isolation of cancer stem cells from adult glioblastoma multiforme. Oncogene 23, 9392-9400

17 Lathia, J.D., et al. (2015) Cancer stem cells in glioblastoma. Genes \& development 29, 12031217

18 Patel, A.P., et al. (2014) Single-cell RNA-seq highlights intratumoral heterogeneity in primary glioblastoma. Science 344, 1396-1401 
19 Wang, Q., et al. (2017) Tumor Evolution of Glioma-Intrinsic Gene Expression Subtypes Associates with Immunological Changes in the Microenvironment. Cancer cell 32, $42-56$

20 Friedmann-Morvinski, D. (2014) Glioblastoma heterogeneity and cancer cell plasticity. Critical reviews in oncogenesis $19,327-336$

21 Albini, A., et al. (2015) Cancer stem cells and the tumor microenvironment: interplay in tumor heterogeneity. Connective Tissue Research 56, 414-425

22 Abou-Antoun, T.J., et al. (2017) Brain Cancer Stem Cells in Adults and Children: Cell Biology and Therapeutic Implications. pp. 372-384

23 Lima FR, K.S., Soletti RC, Biasoli D, Alves T, da Fonseca AC, Garcia C, Romão L, Brito J, Holanda-Afonso R, Faria J, Borges H, Moura-Neto V. (2012) Glioblastoma: therapeutic challenges, what lies ahead. Biochim Biophys Acta 1826, 12

24 Fonseca AC, B.B. (2013) Microglia and Macrophages in Malignant Gliomas: Recent Discoveries and Implications for Promising Therapies. Clinical \& Developmental Immunology 2013, 5 25 Roos, A., et al. (2017) Molecular and Microenvironmental Determinants of Glioma Stem-Like Cell Survival and Invasion. Frontiers in Oncology 7

26 Yin, A.a., et al. (2013) Radiotherapy Plus Concurrent or Sequential Temozolomide for Glioblastoma in the Elderly: A Meta-Analysis. PLOS ONE 8

27 Stupp, R., et al. (2015) GBM Optune/NovoTTF: Maintenance Therapy With Tumor-Treating Fields Plus Temozolomide vs Temozolomide Alone for Glioblastoma: A Randomized Clinical Trial. JAMA 314, 2535-2543

28 Perry, J.R., et al. (2017) Short-Course Radiation plus Temozolomide in Elderly Patients with Glioblastoma. New England Journal of Medicine 376, 1027-1037

29 Friedman, H.S., et al. (2009) Bevacizumab alone and in combination with irinotecan in recurrent glioblastoma. Journal of Clinical Oncology 27, 4733-4740

30 Kreisl, T.N., et al. (2009) Phase II trial of single-agent bevacizumab followed by bevacizumab plus irinotecan at tumor progression in recurrent glioblastoma. Journal of Clinical Oncology 27, 740-745

31 Chamberlain, M.C. and Johnston, S.K. (2010) Salvage therapy with single agent bevacizumab for recurrent glioblastoma. Journal of Neuro-Oncology 96, 259-269

32 Vredenburgh, J.J., et al. (2011) The addition of bevacizumab to standard radiation therapy and temozolomide followed by bevacizumab, temozolomide, and irinotecan for newly diagnosed glioblastoma. Clinical Cancer Research 17, 4119-4124

33 Prosniak, M., et al. (2013) Glioma grade is associated with the accumulation and activity of cells bearing M2 monocyte markers. Clinical Cancer Research 19, 3776-3786

34 Castro, M.G., et al. (2014) Blocking immunosuppressive checkpoints for glioma therapy: The more the merrier! Clinical Cancer Research 20, 5147-5149

35 Ooi, Y.C., et al. (2014) The role of regulatory T-cells in glioma immunology. pp. 125-132 36 Perng, P. and Lim, M. (2015) Immunosuppressive Mechanisms of Malignant Gliomas: Parallels at Non-CNS Sites. Frontiers in oncology 5, 153

37 Quail, D.F. and Joyce, J.A. (2013) Microenvironmental regulation of tumor progression and metastasis. Nat Med 19, 1423-1437

38 Mittal, D., et al. (2014) New insights into cancer immunoediting and its three component phases-elimination, equilibrium and escape. pp. 16-25

39 Emens, L.A., et al. (2017) Cancer immunotherapy: Opportunities and challenges in the rapidly evolving clinical landscape. pp. 116-129

40 Postow, M.A., et al. (2015) Immune checkpoint blockade in cancer therapy. pp. 1974-1982 41 Sharma, P. and Allison, J.P. (2015) Immune checkpoint targeting in cancer therapy: Toward combination strategies with curative potential. Cell 161, 205-214

42 Sharma, P. and Allison, J.P. (2015) The future of immune checkpoint therapy. Science 348, 5661

43 Chamberlain, M.C. and Kim, B.T. (2017) Nivolumab for patients with recurrent glioblastoma progressing on bevacizumab: a retrospective case series. J Neurooncol 133, 561-569 
44 Reiss, S., et al. (2017) Retrospective review of safety and efficacy of checkpoint inhibition in refractory high-grade glioas. Journal of Clinical Oncology 35, 2033-2033

45 Lim, M., et al. (2018) Current state of immunotherapy for glioblastoma. Nat. Rev. Clin. Oncol. doi:10.1038/s41571-018-0003-5 (http://www.nature.com)

46 Ursu, R. and Carpentier, A.F. (2012) Immunotherapeutic approach with oligodeoxynucleotides containing $\mathrm{CpG}$ motifs (CpG-ODN) in malignant glioma. Advances in Experimental Medicine and Biology 746, 95-108

47 El Andaloussi, A., Sonabend, A.M., Han, Y., and Lesniak, M.S. (2006) Stimula-

tion of TLR9 with CpG ODN enhances apoptosis of glioma and prolongs the survival of mice withe xperimental brain tumors. Glia 54,10

$48 \mathrm{Wu}$, A., et al. (2007) In vivo vaccination with tumor cell lysate plus $\mathrm{CpG}$ oligodeoxynucleotides eradicates murine glioblastoma. J Immunother 30, 789-797

49 Alizadeh, D., et al. (2010) Induction of anti-glioma natural killer cell response following multiple low-dose intracerebral CpG therapy. Clinical Cancer Research 16, 3399-3408

$50 \mathrm{Li}$, X., et al. (2012) CpG ODN107 potentiates radiosensitivity of human glioma cells via TLR9mediated NF- $\kappa \mathrm{B}$ activation and NO production. Tumor Biology 33, 1607-1618

$51 \mathrm{Liu}, \mathrm{D}$., et al. (2013) The radiosensitizing effect of CpG ODN107 on human glioma cells is tightly related to its antiangiogenic activity via suppression of HIF-1 $\alpha /$ VEGF pathway.

International Immunopharmacology 17, 237-244

$52 \mathrm{Li}, \mathrm{X}$., et al. (2016) TLR9-ERK-mTOR signaling is critical for autophagic cell death induced by CpG oligodeoxynucleotide 107 combined with irradiation in glioma cells. Scientific reports 6, 27104

53 Jordan, M. and Waxman, D.J. (2016) CpG-1826 immunotherapy potentiates chemotherapeutic and anti-tumor immune responses to metronomic cyclophosphamide in a preclinical glioma model. Cancer Letters 373, 88-96

54 Ouyang, M., et al. (2016) Metronomic doses of temozolomide enhance the efficacy of carbon nanotube CPG immunotherapy in an invasive glioma model. PLOS ONE 11

55 Zhao, D., et al. (2011) Carbon nanotubes enhance CpG uptake and potentiate antiglioma immunity. Clinical Cancer Research 17, 771-782

56 Lollo, G., et al. (2015) Development of multifunctional lipid nanocapsules for the co-delivery of paclitaxel and CpG-ODN in the treatment of glioblastoma. International Journal of Pharmaceutics 495, 972-980

57 Carpentier, A., Laigle-Donadey, F., Zohar, S., Capelle, L., Behin, A., Tibi, A., Martin-

Duverneuil, N., Sanson, M., Lacomblez, L., Taillibert, S., Puybasset, L., Van Effenterre,

R.,Delattre, J.Y., and Carpentier, A.F. (2006) Phase 1 trial of

a CpG oligodeoxynucleotide for patients with recurrent glioblastoma. Neuro Oncol 8, 7

58 Carpentier, A., Metellus, P., Ursu, R., Zohar, S., Lafitte, F., Barrié, M., Meng, Y., Richard, M.,

Parizot, C., Laigle-Donadey, F., Gorochov, G., Psimaras, D., Sanson, M., Tibi, A.,Chinot, O., and

Carpentier, A.F. (2010) Intracere-

bral administration of $\mathrm{CpG}$ oligonucleotide for patients with recurrent glioblastoma:

a phase II study. Neuro Oncol 12,8

59 Ursu, R., et al. (2017) Intracerebral injection of $\mathrm{CpG}$ oligonucleotide for patients with de novo glioblastoma-A phase II multicentric, randomised study. European Journal of Cancer 73, 30-37 60 Lollini, P.-L., et al. (2006) Vaccines for tumour prevention. Nature Reviews Cancer 6, 204-216 61 Aldape, K.D., et al. (2004) Immunohistochemical Detection of EGFRvIII in High Malignancy Grade Astrocytomas and Evaluation of Prognostic Significance. Journal of Neuropathology and Experimental Neurology 63, 700-707

62 Heimberger, A.B., et al. (2005) Prognostic effect of epidermal growth factor receptor and EGFRvIII in glioblastoma multiforme patients. Clinical cancer research : an official journal of the American Association for Cancer Research 11, 1462-1466

63 Libermann, T.A., et al. (1985) Amplification, enhanced expression and possible rearrangement of EGF receptor gene in primary human brain tumours of glial origin. Nature 313, 144-147 
64 Nagane, M., et al. (1996) A common mutant epidermal growth factor receptor confers enhanced tumorigenicity on human glioblastoma cells by increasing proliferation and reducing apoptosis.

Cancer Research 56, 5079-5086

65 Lammering, G., et al. (2003) EGFRvIII-mediated radioresistance through a strong cytoprotective response. Oncogene 22, 5545-5553

66 Heimberger, A.B., et al. (2003) Epidermal growth factor receptor VIII peptide vaccination is efficacious against established intracerebral tumors. Clinical Cancer Research 9, 4247-4254

67 Sampson, J.H., et al. (2010) Immunologic escape after prolonged progression-free survival with epidermal growth factor receptor variant III peptide vaccination in patients with newly diagnosed glioblastoma. Journal of Clinical Oncology 28, 4722-4729

68 Sampson, J.H., et al. (2011) Greater chemotherapy-induced lymphopenia enhances tumorspecific immune responses that eliminate EGFRvIII-expressing tumor cells in patients with glioblastoma. Neuro-Oncology 13, 324-333

69 Schuster, J., et al. (2015) A phase II, multicenter trial of rindopepimut (CDX-110) in newly diagnosed glioblastoma: The ACT III study. Neuro-Oncology 17, 854-861

70 Weller, M., et al. (2017) Rindopepimut with temozolomide for patients with newly diagnosed, EGFRvIII-expressing glioblastoma (ACT IV): a randomised, double-blind, international phase 3 trial. Lancet Oncol 18, 1373-1385

71 Maus, M.V., et al. (2014) Antibody-modified T cells: CARs take the front seat for hematologic malignancies. Blood 123, 2625

72 Priceman, S.J., et al. (2015) Smart CARs engineered for cancer immunotherapy. Current Opinion in Oncology 27, 466-474

73 Jackson, H.J., et al. (2016) Driving CAR T-cells forward. Nature Reviews Clinical Oncology 13, 370-383

74 Morgan, R.A., et al. (2012) Recognition of Glioma Stem Cells by Genetically Modified T Cells Targeting EGFRvIII and Development of Adoptive Cell Therapy for Glioma. Human Gene Therapy 23, 1043-1053

75 Johnson, L.A., et al. (2015) Rational development and characterization of humanized anti-EGFR variant III chimeric antigen receptor T cells for glioblastoma. Science Translational Medicine 7 , $275 \mathrm{ra} 222$

76 O'Rourke, D.M., et al. (2017) A single dose of peripherally infused EGFRvIII-directed CAR T cells mediates antigen loss and induces adaptive resistance in patients with recurrent glioblastoma. Science Translational Medicine 9, eaaa0984-eaaa0984

77 Ahmed, N., et al. (2010) HER2-specific T cells target primary glioblastoma stem cells and induce regression of autologous experimental tumors. Clinical Cancer Research 16, 474-485

78 Brown, C.E., et al. (2012) Stem-like tumor-initiating cells isolated from IL13R??2 expressing gliomas are targeted and killed by IL13-zetakine-redirected T cells. Clinical Cancer Research 18, 2199-2209

79 Chow, K.K.H., et al. (2013) T Cells Redirected to EphA2 for the Immunotherapy of Glioblastoma. Molecular Therapy 21, 629-637

80 Brown, C.E., et al. (2015) Bioactivity and Safety of IL13R $\alpha 2$-Redirected Chimeric Antigen Receptor CD8+ T Cells in Patients with Recurrent Glioblastoma. pp. 4062-4072

81 Brown, C.E., et al. (2016) Regression of Glioblastoma after Chimeric Antigen Receptor T-Cell Therapy. New England Journal of Medicine 375, 2561-2569

82 Lawler, S.E. and Chiocca, E.A. (2015) Oncolytic Virus-Mediated Immunotherapy: A Combinatorial Approach for Cancer Treatment. Journal of Clinical Oncology 33, 2812-2814

83 Chiocca, E.A. and Rabkin, S.D. (2014) Oncolytic Viruses and Their Application to Cancer Immunotherapy. Cancer immunology research 2, 295-300

84 Angelova, A.L., et al. (2017) Immunotherapeutic Potential of Oncolytic H-1 Parvovirus: Hints of Glioblastoma Microenvironment Conversion towards Immunogenicity. Viruses 9, 382

85 Geletneky, K., et al. (2017) Oncolytic H-1 Parvovirus Shows Safety and Signs of Immunogenic Activity in a First Phase I/IIa Glioblastoma Trial. Molecular Therapy 25, 2620-2634 
86 Qiao, J., et al. (2015) Intratumoral oncolytic adenoviral treatment modulates the glioma microenvironment and facilitates systemic tumor-antigen-specific T cell therapy. Oncoimmunology 4, e1022302

87 Brown, M.C., et al. (2017) Cancer immunotherapy with recombinant poliovirus induces IFNdominant activation of dendritic cells and tumor antigen-specific CTLs. Science Translational Medicine 9, eaan4220

88 Jain RK, d.T.E., Duda DG, Loeffler JS, Sorensen AG, Batchelor TT. (2007) Angiogenesis in brain tumours. Nat Rev Neurosci. 8, 13

89 Ricci-Vitiani L, P.R., Biffoni M, Todaro M, Invernici G, Cenci T, Maira G, Parati EA, Stassi G, Larocca LM, De Maria R (2010) Tumour vascularization via endothelial differentiation of glioblastoma stem-like cells. Nature 468, 5

90 Daumas-Duport, C. (1992) Histological grading of gliomas. Curr Opin Neurol Neurosurg 5, 924-931

91 Mathivet, T., et al. (2017) Dynamic stroma reorganization drives blood vessel dysmorphia during glioma growth. EMBO Molecular Medicine 9, 1629-1645

92 Jain, R.K. (2005) Normalization of tumor vasculature: an emerging concept in antiangiogenic therapy. Science 307, 58-62

93 Takano, S., et al. (2014) Bevacizumab in Japanese patients with malignant glioma: From basic research to clinical trial. OncoTargets and Therapy 7, 1551-1562

94 Tamura, R., et al. (2016) Histopathological investigation of glioblastomas resected under bevacizumab treatment. Oncotarget 7

95 Gilbert, M.R., et al. (2014) A randomized trial of bevacizumab for newly diagnosed glioblastoma. The New England journal of medicine 370, 699-708

96 Gil-Gil, M., et al. (2013) Bevacizumab for the treatment of Glioblastoma. Clin Med Insights

Oncol. 7, 123-135

97 Tamura, R., et al. (2017) Bevacizumab for malignant gliomas: current indications, mechanisms of action and resistance, and markers of response. Brain Tumor Pathology 34, 62-77

98 Thompson, E.M., et al. (2011) The paradoxical effect of bevacizumab in the therapy of malignant gliomas. Neurology 76, 87-93

99 Lu, K.V., et al. (2012) VEGF Inhibits Tumor Cell Invasion and Mesenchymal Transition through a MET/VEGFR2 Complex. Cancer Cell 22, 21-35

100 Huang, M., et al. (2016) c-Met-mediated endothelial plasticity drives aberrant vascularization and chemoresistance in glioblastoma. The Journal of Clinical Investigation 126, 1801-1814

101 Labussière, M., et al. (2016) Angiopoietin-2 May Be Involved in the Resistance to Bevacizumab in Recurrent Glioblastoma. Cancer Investigation 7907, 1-6

102 Tabouret, E., et al. (2016) Changes in PIGF and MET-HGF expressions in paired initial and recurrent glioblastoma. Journal of Neuro-Oncology 130, 431-437

103 Carmeliet, P., et al. (2001) Synergism between vascular endothelial growth factor and placental growth factor contributes to angiogenesis and plasma extravasation in pathological conditions.

Nature Medicine 7, 575-583

104 Lassen, U., et al. (2015) Phase 1 dose-escalation study of the antiplacental growth factor monoclonal antibody RO5323441 combined with bevacizumab in patients with recurrent glioblastoma.

Neuro-Oncology 17, 1007-1015

105 Erdem-Eraslan, L., et al. (2016) Identification of patients with recurrent glioblastoma who may benefit from combined bevacizumab and CCNU Therapy: A Report from the BELOB Trial. Cancer Research 76, 525-534

106 Weathers, S.P., et al. (2016) A randomized phase II trial of standard dose bevacizumab versus low dose bevacizumab plus lomustine (CCNU) in adults with recurrent glioblastoma. J Neurooncol $129,487-494$

107 Wick, W., et al. (2010) Phase III study of enzastaurin compared with lomustine in the treatment of recurrent intracranial glioblastoma. Journal of Clinical Oncology 28, 1168-1174 
108 Levin, V.A., et al. (2017) Effect of angiotensin system inhibitors on survival in newly diagnosed glioma patients and recurrent glioblastoma patients receiving chemotherapy and/or bevacizumab. J Neurooncol 134, 325-330

109 Carpentier, A.F., et al. (2012) Steroid-sparing effects of angiotensin-II inhibitors in glioblastoma patients. European Journal of Neurology 19, 1337-1342

110 Januel, E., et al. (2015) Impact of renin-angiotensin system blockade on clinical outcome in glioblastoma. European Journal of Neurology 22, 1304-1309

111 Arrieta, O., et al. (2005) Blockage of angiotensin II type I receptor decreases the synthesis of growth factors and induces apoptosis in C6 cultured cells and C6 rat glioma. British journal of cancer $92,1247-1252$

112 Kourilsky, A., et al. (2016) Impact of Angiotensin-II receptor blockers on vasogenic edema in glioblastoma patients. Journal of Neurology 263, 524-530

113 Wong, M.L.H., et al. (2009) Tumour angiogenesis: Its mechanism and therapeutic implications in malignant gliomas. Journal of Clinical Neuroscience 16, 1119-1130

114 Gerstner, E.R., et al. (2011) Phase I trial with biomarker studies of vatalanib (PTK787) in patients with newly diagnosed glioblastoma treated with enzyme inducing anti-epileptic drugs and standard radiation and temozolomide. Journal of neuro-oncology 103, 325-332

115 Chi, A.S., et al. (2009) Antiangiogenic strategies for treatment of malignant gliomas. Neurotherapeutics 6, 513-526

116 Wick, W., et al. (2016) Current status and future directions of anti-angiogenic therapy for gliomas. Neuro-Oncology 18, 315-328

117 Zhang, M., et al. (2015) Recent advance in molecular angiogenesis in glioblastoma: the challenge and hope for anti-angiogenic therapy. Brain Tumor Pathology 32, 229-236

118 Gruslova, A., et al. (2015) VB-111: a novel anti-vascular therapeutic for glioblastoma multiforme. Journal of Neuro-Oncology 124, 365-372

119 Kushchayev, S.V., et al. (2014) Monocyte-derived cells of the brain and malignant gliomas: The double face of janus. pp. 1171-1186

120 Nishie, A., et al. (1999) Macrophage Infiltration and Heme Oxygenase-1 Expression Correlate with Angiogenesis in Human Gliomas. Clinical Cancer Research 5, 1107-1113

121 Pyonteck, S.M., et al. (2013) CSF-1R inhibition alters macrophage polarization and blocks glioma progression. Nat Med 19, 1264-1272

122 Saijo, K. and Glass, C.K. (2011) Microglial cell origin and phenotypes in health and disease.

Nat. Rev. Immunol. 11, 775-787

123 Mosser, D.M. and Edwards, J.P. (2008) Exploring the full spectrum of macrophage activation.

Nat. Rev. Immunol. 8, 958-969

124 Franco, R. and Fernández-Suárez, D. (2015) Alternatively activated microglia and macrophages in the central nervous system. Progress in Neurobiology 131, 65-86

125 Cinzia Dello, R., et al. (2017) Exploiting Microglial Functions for the Treatment of Glioblastoma. Current Cancer Drug Targets 17, 267-281

$126 \mathrm{Yu}$, H., Kortylewski, M., and Pardoll, D. (2007) Crosstalk between cancer and immune cells: role of STAT3 in the tumour microenvironment. Nat Rev Immunol. 7, 11

127 Bromberg, J., and Wang, T.C. (2009) Inflammation and cancer: IL-6 and STAT3 complete the link. Cancer Cell 15, 12

128 Zhang, L., Alizadeh, D., Van Handel, M., Kortylewski, M., Yu, H., and Badie, B. (2009) Stat3 inhibition activates tumormacrophages and abrogates glioma growth in mice. Glia 57, 12

129 Zhang, L., Liu, W., Alizadeh, D., Zhao, D., Farrukh, O., Lin, J., Badie, S.A., and Badie, B. (2009) S100B Attenuates Microglia Activation in Gliomas: Possible Role of STAT3 Pathway. Glia 59,13

130 Cheng, F., Wang, H.W., Cuenca, A., Huang, M., Ghansah, T., Brayer, J., Kerr, W.G., Takeda, K., Akira, S., Schoenberger, S.P., Yu, H., Jove, R., and Sotomayor, E.M. (2003) A critical role for Stat3 signaling in immune tolerance. Immunity 19, 12 
131 Hussain, S.F., Kong, L.Y., Jordan, J., Conrad, C., Madden, T., Fokt, I., Priebe, W., and Heimberger, A.B. (2007)

A novel small molecule inhibitor of signal transducers and activators of transcription 3 reverses im munetolerance in malignant glioma patients. Cancer Res 67, 7

132 Iwamaru, A., Szymanski, S., Iwado, E., Aoki, H., Yokoyama, T., Fokt, I., Hess, K., Conrad, C., Madden, T., Sawaya, R., Kondo, S., Priebe, W., and Kondo, Y. (2007) A novel inhibitor of the STAT3 pathway induces apoptosis in malignant glioma cells both in vitro and in vivo. Oncogene 26,10

133 Lang, R., Patel, D., Morris, J.J., Rutschman, R.L., and Murray, P.J. (2002) Shaping gene expression in activated and resting primary macrophages by IL-10. J Immunol. 169, 11

134 Sansone, P., and Bromberg, J. (2012) Targeting the interleukin-

6/Jak/stat pathway in human malignancies. J Clin Oncol. 30, 10

135 Michaylira, C.Z., et al. (2010) Periostin, a cell adhesion molecule, facilitates invasion in the tumor microenvironment and annotates a novel tumor-invasive signature in esophageal cancer.

Cancer Research 70, 5281-5292

136 Guo, X., et al. (2016) Hypoxia promotes glioma-associated macrophage infiltration via periostin and subsequent M2 polarization by upregulating TGF-beta and M-CSFR. Oncotarget 7, 8052180542

137 Shen, X., et al. (2016) Glioma-induced inhibition of caspase-3 in microglia promotes a tumorsupportive phenotype. Nature Immunology 17, 1282-1290

138 Coniglio, S.J., et al. (2012) Microglial Stimulation of Glioblastoma Invasion Involves Epidermal Growth Factor Receptor (EGFR) and Colony Stimulating Factor 1 Receptor (CSF-1R) Signaling. Molecular Medicine 18, 519-527

139 Fixe, P. and Praloran, V. (1997) Macrophage colony-stimulating-factor (M-CSF or CSF-1) and its receptor: structure-function relationships. European Cytokine Network 8, 125-136

140 Quail, D.F., et al. (2016) The tumor microenvironment underlies acquired resistance to CSF1R inhibition in gliomas. Science (New York, N.Y.) 352, aad3018

141 Butowski, N., et al. (2016) Orally administered colony stimulating factor 1 receptor inhibitor PLX3397 in recurrent glioblastoma: an Ivy Foundation Early Phase Clinical Trials Consortium phase II study. Neuro-Oncology 18, 557-564

142 Rodero, M., et al. (2008) Polymorphism in the microglial cell-mobilizing CX3CR1 gene is associated with survival in patients with glioblastoma. Journal of Clinical Oncology 26, 5957-5964 143 Bowman, R.L., et al. (2016) Macrophage ontogeny underlies differences in tumor-specific education in brain malignancies. Cell reports 17, 2445-2459

144 Ricard, C., et al. (2016) Phenotypic dynamics of microglial and monocyte-derived cells in glioblastoma-bearing mice. Scientific Reports 6, 26381

145 Wang, Q., et al. (2018) Vascular niche IL-6 induces alternative macrophage activation in glioblastoma through HIF-2 $\alpha$. Nature Commun. 9, 559

146 Hanahan, D. and Weinberg, R.A. (2011) Hallmarks of cancer: The next generation. pp. 646674

147 Ward, P.S. and Thompson, C.B. (2012) Metabolic Reprogramming: A Cancer Hallmark Even Warburg Did Not Anticipate. pp. 297-308

148 Warburg, O. (1956) Injuring of Respiration the Origin of Cancer Cells. Science 123, 309-314 149 Vander Heiden, M.G., et al. (2009) Understanding the Warburg Effect: The Metabolic Requirements of Cell Proliferation. Science 324, 1029-1033

150 Cairns, R.A., et al. (2011) Regulation of cancer cell metabolism. Nature Reviews Cancer 11, $85-95$

151 Kessler, R., et al. (2008) 6-Phosphofructo-2-kinase/fructose-2,6-bisphosphatase (PFKFB3) is up-regulated in high-grade astrocytomas. Journal of Neuro-Oncology 86, 257-264

152 Wolf, A., et al. (2011) Hexokinase 2 is a key mediator of aerobic glycolysis and promotes tumor growth in human glioblastoma multiforme. The Journal of Experimental Medicine 208, 313326 
153 Flavahan, W.A., et al. (2013) Brain tumor initiating cells adapt to restricted nutrition through preferential glucose uptake. Nature Neuroscience 16, 1373-1382

154 Vartanian, A., et al. (2016) Targeting Hexokinase 2 enhances response to radio-chemotherapy in Glioblastoma. Oncotarget 7, 69518-69535

155 Kefas, B., et al. (2010) Pyruvate kinase M2 is a target of the tumorsuppressive microRNA-326 and regulates the survival of glioma cells. Neuro-Oncology 12, 1102-1112

156 Mukherjee, J., et al. (2013) Pyruvate Kinase M2 Expression, but Not Pyruvate Kinase Activity, Is Up-Regulated in a Grade-Specific Manner in Human Glioma. PLoS ONE 8

157 Ros, S. and Schulze, A. (2013) Balancing Glycolytic Flux: The Role of 6-Phosphofructo-2-

Kinase/fructose 2,6-Bisphosphatases in Cancer Metabolism. Cancer \& Metabolism 1, 8-16

158 Rodríguez-Garcia, A., et al. (2017) TGF- $\beta 1$ Targets Smad, p38 MAPK, and PI3K/Akt Signaling Pathways to Induce PFKFB3 Gene Expression and Glycolysis in Glioblastoma Cells. The FEBS Journal 284, 3437-3454

159 Goidts, V., et al. (2012) RNAi screening in glioma stem-like cells identifies PFKFB4 as a key molecule important for cancer cell survival. Oncogene 31, 3235-3243

160 Sborov, D.W., et al. (2015) Investigational Cancer Drugs Targeting Cell Metabolism in Clinical Development. Expert Opinion on Investigational Drugs 24, 79-94

161 Pelicano, H., et al. (2006) Glycolysis inhibition for anticancer treatment. Oncogene 25, 46334646

162 Oudard, S., et al. (2003) Phase II study of lonidamine and diazepam in the treatment of recurrent glioblastoma multiforme. Journal of Neuro-Oncology 63, 81-86

163 Michelakis, E.D., et al. (2008) Dichloroacetate (DCA) as a potential metabolic-targeting therapy for cancer. British Journal of Cancer 99, 989-994

164 Yan, H., et al. (2009) IDH1 and IDH2 Mutations in Gliomas. New England Journal of Medicine 360, 765-773

165 Zhao, S., et al. (2009) Glioma-derived mutations in IDH1 dominantly inhibit IDH1 catalytic activity and induce HIF-1alpha. Science (New York, N.Y.) 324, 261-265

166 Chowdhury, R., et al. (2011) The oncometabolite 2-hydroxyglutarate inhibits histone lysine demethylases. EMBO reports 12, 463-469

$167 \mathrm{Xu}, \mathrm{W}$., et al. (2011) Oncometabolite 2-hydroxyglutarate is a competitive inhibitor of $\alpha$ ketoglutarate-dependent dioxygenases. Cancer Cell 19, 17-30

168 Chen, J., et al. (2016) The Evolving Landscape in the Development of Isocitrate Dehydrogenase Mutant Inhibitors. Mini Rev Med Chem 16, 1344-1358

169 Pusch, S., et al. (2017) Pan-mutant IDH1 inhibitor BAY 1436032 for effective treatment of IDH1 mutant astrocytoma in vivo. Acta Neuropathologica 133, 629-644

170 Yen, K., et al. (2017) AG-221, a First-in-Class Therapy Targeting Acute Myeloid Leukemia Harboring Oncogenic IDH2 Mutations. Cancer Discovery 7, 478-493

171 Oudard, S., et al. (1996) High glycolysis in gliomas despite low hexokinase transcription and activity correlated to chromosome 10 loss. British journal of cancer 74, 839-845

172 Tanaka, K., et al. (2015) Compensatory glutamine metabolism promotes glioblastoma resistance to mTOR inhibitor treatment. Journal of Clinical Investigation 125, 1591-1602

173 Jelluma, N., et al. (2006) Glucose withdrawal induces oxidative stress followed by apoptosis in glioblastoma cells but not in normal human astrocytes. Molecular cancer research : MCR 4, 319330

174 Derr, R.L., et al. (2009) Association between hyperglycemia and survival in patients with newly diagnosed glioblastoma. Journal of Clinical Oncology 27, 1082-1086

175 Tieu, M.T., et al. (2015) Impact of glycemia on survival of glioblastoma patients treated with radiation and temozolomide. Journal of Neuro-Oncology 124, 119-126

176 Chang, H.T., et al. (2013) Ketolytic and Glycolytic Enzymatic Expression Profiles in Malignant Gliomas: Implication for Ketogenic Diet Therapy. Nutrition \& Metabolism 10, 47-57

177 Winter, S.F., et al. (2017) Role of ketogenic metabolic therapy in malignant glioma: A systematic review. pp. 41-58 
178 Strickland, M. and Stoll, E.A. (2017) Metabolic Reprogramming in Glioma. Frontiers in Cell and Developmental Biology 5

179 Esteller, M. (2011) Non-coding RNAs in human disease. Nat. Rev. Genet. 12, 861-874

180 Bartel, D.P. (2004) MicroRNAs: Genomics, Biogenesis, Mechanism, and Function. Cell 116, 281-297

181 Hanke, M., et al. (2010) A robust methodology to study urine microRNA as tumor marker: MicroRNA-126 and microRNA-182 are related to urinary bladder cancer. Urologic Oncology: Seminars and Original Investigations 28, 655-661

182 Toiyama, Y., et al. (2013) Serum miR-21 as a diagnostic and prognostic biomarker in colorectal cancer. Journal of the National Cancer Institute 105, 849-859

183 Fareh, M., et al. (2017) Cell-based therapy using miR-302-367 expressing cells represses glioblastoma growth. Cell Death and Disease 8, e2713-e2713

$184 \mathrm{Xu}, \mathrm{L}$., et al. (2017) MiR-543 functions as a tumor suppressor in glioma in vitro and in vivo. Oncology Reports 38, 725-734

185 Gabriely, G., et al. (2011) Human glioma growth is controlled by microRNA-10b. Cancer Research 71, 3563-3572

186 Guessous, F., et al. (2013) Oncogenic effects of miR-10b in glioblastoma stem cells. Journal of Neuro-Oncology 112, 153-163

187 Zhou, X., et al. (2010) Downregulation of miR-21 inhibits EGFR pathway and suppresses the growth of human glioblastoma cells independent of PTEN status. Laboratory investigation; a journal of technical methods and pathology 90, 144-155

$188 \mathrm{Kim}, \mathrm{H}$., et al. (2010) Integrative genome analysis reveals an oncomir/oncogene cluster regulating glioblastoma survivorship. Proceedings of the National Academy of Sciences 107, 2183-2188

189 Kefas, B., et al. (2008) microRNA-7 inhibits the epidermal growth factor receptor and the akt pathway and is down-regulated in glioblastoma. Cancer Research 68, 3566-3572

190 Rathod, S.S., et al. (2014) Tumor suppressive miRNA-34a suppresses cell proliferation and tumor growth of glioma stem cells by targeting Akt and Wnt signaling pathways. FEBS Open Bio 4, 485-495

$191 \mathrm{Gu}$, J.J., et al. (2015) MiR-218 inhibits the migration and invasion of glioma u87 cells through the slit2-robo1 pathway. Oncology Letters 9, 1561-1566

192 Wang, R.J., et al. (2015) MicroRNA-873 (MiRNA-873) inhibits glioblastoma tumorigenesis and metastasis by suppressing the expression of IGF2BP1. Journal of Biological Chemistry 290, 8938-8948

193 Huang, T., et al. (2013) Noncoding RNAs in cancer and cancer stem cells. Chinese journal of cancer 32, 582-593

194 Takahashi, R.-u., et al. (2014) The role of microRNAs in the regulation of cancer stem cells. Frontiers in Genetics 4

195 Shi, L., et al. (2010) MiR-125b is critical for the suppression of human U251 glioma stem cell proliferation. Brain Research 1312, 120-126

196 Schraivogel, D., et al. (2011) CAMTA1 is a novel tumour suppressor regulated by miR-9/9 $<$ sup $>*<$ sup > in glioblastoma stem cells. The EMBO Journal 30, 4309-4322

197 Zhao, S., et al. (2013) MiR-143 inhibits glycolysis and depletes stemness of glioblastoma stemlike cells. Cancer Letters 333, 253-260

198 Areeb, Z., et al. (2015) MicroRNA as potential biomarkers in Glioblastoma. pp. 237-248

199 Rivera-Díaz, M., et al. (2015) MicroRNA-27a distinguishes glioblastoma multiforme from diffuse and anaplastic astrocytomas and has prognostic value. American journal of cancer research 5, 201-218

200 Barbano, R., et al. (2014) A miRNA signature for defining aggressive phenotype and prognosis in gliomas. PLOS ONE 9

201 Visani, M., et al. (2014) Expression of 19 microRNAs in glioblastoma and comparison with other brain neoplasia of grades I-III. Molecular Oncology 8, 417-430 
202 Lozada-Delgado, E.L., et al. (2017) RNA interference for glioblastoma therapy: Innovation ladder from the bench to clinical trials. pp. 26-36

$203 \mathrm{Ma}$, S., et al. (2015) Activating transcription factor 3 is overexpressed in human glioma and its knockdown in glioblastoma cells causes growth inhibition both in vitro and in vivo. Int J Mol Med $35,1561-1573$

204 Gillespie, D.L., et al. (2015) RNA interference targeting hypoxia-inducible factor $1 \alpha$ via a novel multifunctional surfactant attenuates glioma growth in an intracranial mouse model. Journal of Neurosurgery 122, 331-341

205 Frömberg, A., et al. (2017) Analysis of cellular and molecular antitumor effects upon inhibition of SATB1 in glioblastoma cells. BMC Cancer 17, 3-12

206 Tan, S.J., et al. (2011) Engineering nanocarriers for siRNA delivery. pp. 841-856

207 Omar, A.I. and Mason, W.P. (2009) Temozolomide: The evidence for its therapeutic efficacy in malignant astrocytomas. pp. 93-111

208 Taphoorn, M.J.B., et al. (2015) Health-related quality of life in a randomized phase III study of bevacizumab, temozolomide, and radiotherapy in newly diagnosed glioblastoma. Journal of Clinical Oncology 33, 2166-2175

209 Fan, C.H., et al. (2013) O6-methylguanine DNA methyltransferase as a promising target for the treatment of temozolomide-resistant gliomas. Cell death \& disease 4, e876-e876

210 Borhani, S., et al. (2017) In vitro radiosensitizing effects of temozolomide on U87MG cell lines of human glioblastoma multiforme. Iranian Journal of Medical Sciences 42, 258-265

211 Lee, S.Y. (2016) Temozolomide resistance in glioblastoma multiforme. PLoS One 3, 198-210

212 Gerson, S.L. (2004) MGMT: its role in cancer aetiology and cancer therapeutics. Nature reviews. Cancer 4, 296-307

213 Hegi, M.E., et al. (2008) Correlation of O6-methylguanine methyltransferase (MGMT) promoter methylation with clinical outcomes in glioblastoma and clinical strategies to modulate

MGMT activity. pp. 4189-4199

214 Kirson, E.D., et al. (2004) Disruption of Cancer Cell Replication by Alternating Electric Fields. Cancer Research 64, 3288-3295

215 Kirson, E.D., et al. (2007) Alternating electric fields arrest cell proliferation in animal tumor models and human brain tumors. Proceedings of the National Academy of Sciences 104, 1015210157

216 Swanson, K.D., et al. (2016) An Overview of Alternating Electric Fields Therapy (NovoTTF Therapy) for the Treatment of Malignant Glioma. pp. 1-10

217 Kirson, E.D., et al. (2009) Alternating electric fields (TTFields) inhibit metastatic spread of solid tumors to the lungs. Clinical and Experimental Metastasis 26, 633-640

218 Kirson, E.D., et al. (2009) Chemotherapeutic treatment efficacy and sensitivity are increased by adjuvant alternating electric fields (TTFields). BMC medical physics 9,1

219 Lacouture, M.E., et al. (2014) Characterization and management of dermatologic adverse events with the NovoTTF-100A system, a novel anti-mitotic electric field device for the treatment of recurrent glioblastoma. Seminars in Oncology 41

220 Kesari, S.H., and Ram, Z., on behalf of EF-14 Trial Investigators. (2017) Tumor-treating fields plus chemotherapy versus chemotherapy alone for glioblastoma at first recurrence: a post hoc analysis of the EF-14 trial. CNS Oncol 6, 185-193

221 Bernard-Arnoux, F., et al. (2016) The cost-effectiveness of tumor-treating fields therapy in patients with newly diagnosed glioblastoma. Neuro-Oncology 18, 1129-1136 222 Moitra, K. (2015) Overcoming Multidrug Resistance in Cancer Stem Cells. BioMed Research International 2015, 8

223 Gottesman, M.M. (2002) Mechanisms of Cancer Drug Resistance. Annual Review of Medicine $53,615-627$

224 Hall, M.D., et al. (2009) Is resistance useless? Multidrug resistance and collateral sensitivity. pp. 546-556 
225 Dantzig, A.H., et al. (2004) Evaluation of the binding of the tricyclic isoxazole photoaffinity label LY475776 to multidrug resistance associated protein 1 (MRP1) orthologs and several ATPbinding cassette (ABC) drug transporters. Biochemical Pharmacology 67, 1111-1121

226 Stavrovskaya, A.A., et al. (2016) Problems of Glioblastoma Multiforme Drug Resistance. Biochemistry. Biokhimiiā $81,91-100$

227 Tivnan, A., et al. (2015) Inhibition of multidrug resistance protein 1 (MRP1) improves chemotherapy drug response in primary and recurrent glioblastoma multiforme. Frontiers in Neuroscience 9

228 Scotto, K.W. (2003) Transcriptional regulation of ABC drug transporters. Oncogene 22, 74967511

229 Munoz, J.L., et al. (2014) Temozolomide induces the production of epidermal growth factor to regulate MDR1 expression in glioblastoma cells. Mol Cancer Ther 13, 2399-2411

230 Munoz, J.L., et al. (2015) Temozolomide competes for P-glycoprotein and contributes to chemoresistance in glioblastoma cells. Cancer Letters 367, 69-75

231 Muni, R., et al. (2010) Short-term radiotherapy followed by adjuvant chemotherapy in poorprognosis patients with glioblastoma. Tumori 96, 60-64

232 Hosokawa, Y., et al. (2015) Oct-3/4 modulates the drug-resistant phenotype of glioblastoma cells through expression of ATP binding cassette transporter G2. Biochim Biophys Acta 1850, 11971205

233 Auffinger, B., et al. (2013) REVIEW: Drug-Loaded Nanoparticle Systems And Adult Stem Cells: A Potential Marriage For The Treatment Of Malignant Glioma? Oncotarget 4, 378-396 234 Fang, C., et al. (2015) Temozolomide Nanoparticles for Targeted Glioblastoma Therapy. ACS applied materials \& interfaces 7, 6674-6682

$235 \mathrm{Kim}$, S.S., et al. (2015) Encapsulation of temozolomide in a tumor-targeting nanocomplex enhances anti-cancer efficacy and reduces toxicity in a mouse model of glioblastoma. Cancer Letters $369,250-258$

236 Huang, Y., et al. (2016) c(RGDyK)-decorated Pluronic micelles for enhanced doxorubicin and paclitaxel delivery to brain glioma. International Jounal of Nanomedicine 11, 1629-1641

237 Wang, G., et al. (2016) In vitro and in vivo evaluation of functionalized chitosan-Pluronic micelles loaded with myricetin on glioblastoma cancer. Nanomedicine: Nanotechnology, Biology, and Medicine 12, 1263-1278

238 Bobo, R.H., et al. (1994) Convection-enhanced delivery of macromolecules in the brain. Proc Natl Acad Sci U S A 91, 2076-2080

239 Vargová, L., et al. (2003) Diffusion parameters of the extracellular space in human gliomas. Glia 42, 77-88

240 Raghavan, R., et al. (2006) Convection-enhanced delivery of therapeutics for brain disease, and its optimization. Neurosurgical focus 20, E12-E12

241 Jahangiri, A., et al. (2016) Convection-enhanced delivery in glioblastoma: a review of preclinical and clinical studies. Journal of neurosurgery, 1-10

242 Leuthardt, E.C., et al. (2016) Hyperthermic Laser Ablation of Recurrent Glioblastoma Leads to Temporary Disruption of the Peritumoral Blood Brain Barrier. PloS one 11, e0148613-e0148613

\section{Highlights:}

- Glioblastoma remains an incurable disease with a poor prognosis, despite being among the most studied tumors in the past decade 
- About a third of the tumor mass is composed of non-neoplasic infiltrating cells, reflecting a very heterogeneous tumor both in terms of pathology and microenvironment.

- The heterogeneous microenvironment turns Glioblastoma into a good candidate to cell and immunotherapies. But it also constitutes a challenge for interpreting treatment-response and developing new strategies.

\section{Outstanding Questions:}

- Will CAR-T cell therapy prove its way and become the first successful immunotherapy for GBM, by overcoming the highly immunosuppressive microenvironment?

- How can we effectively disrupt GBM interaction with infiltrating non-tumoral cells (such as microglia/macrophages and endothelial cells)?

- Is there a role for anti-angiogenic therapy in GBM or will it remain with symptomatic use of VEGF inhibitors?

- Can metabolic alterations be used as biomarkers for distinguishing tumor subtypes or used as therapeutic targets? 


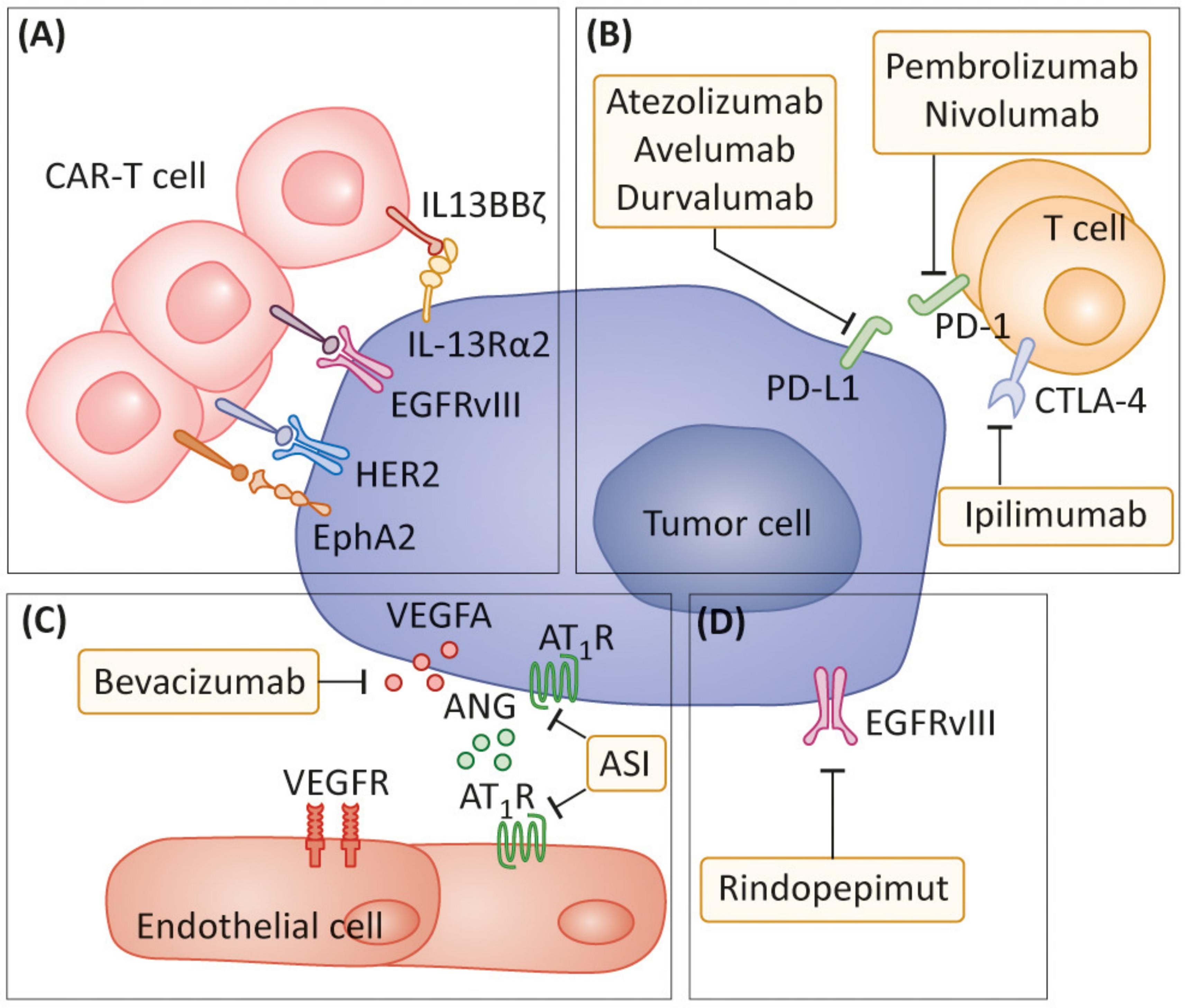


(A) IBA

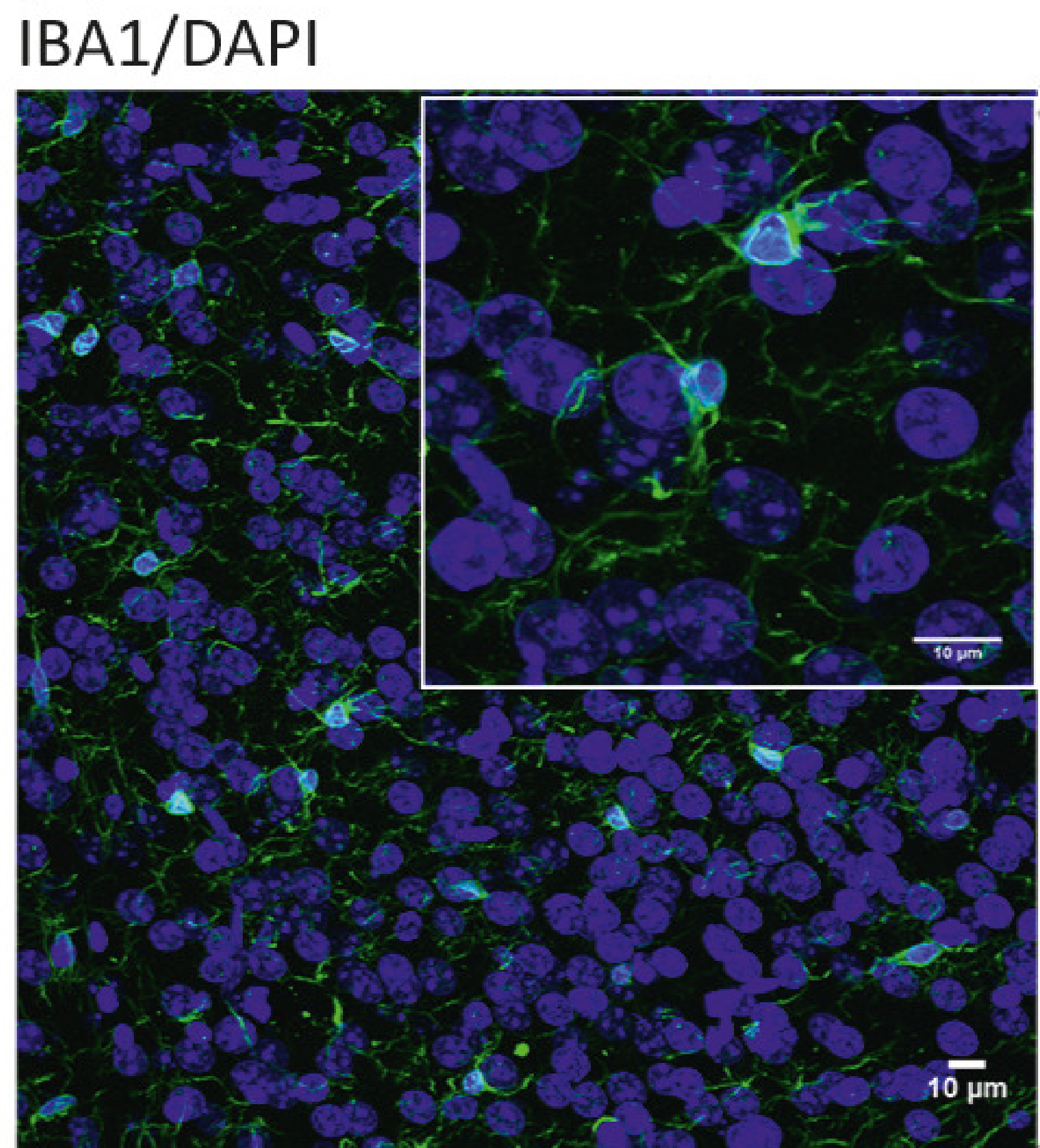

$50 \times 10^{4} \mathrm{GBM}$ cells into swiss brain

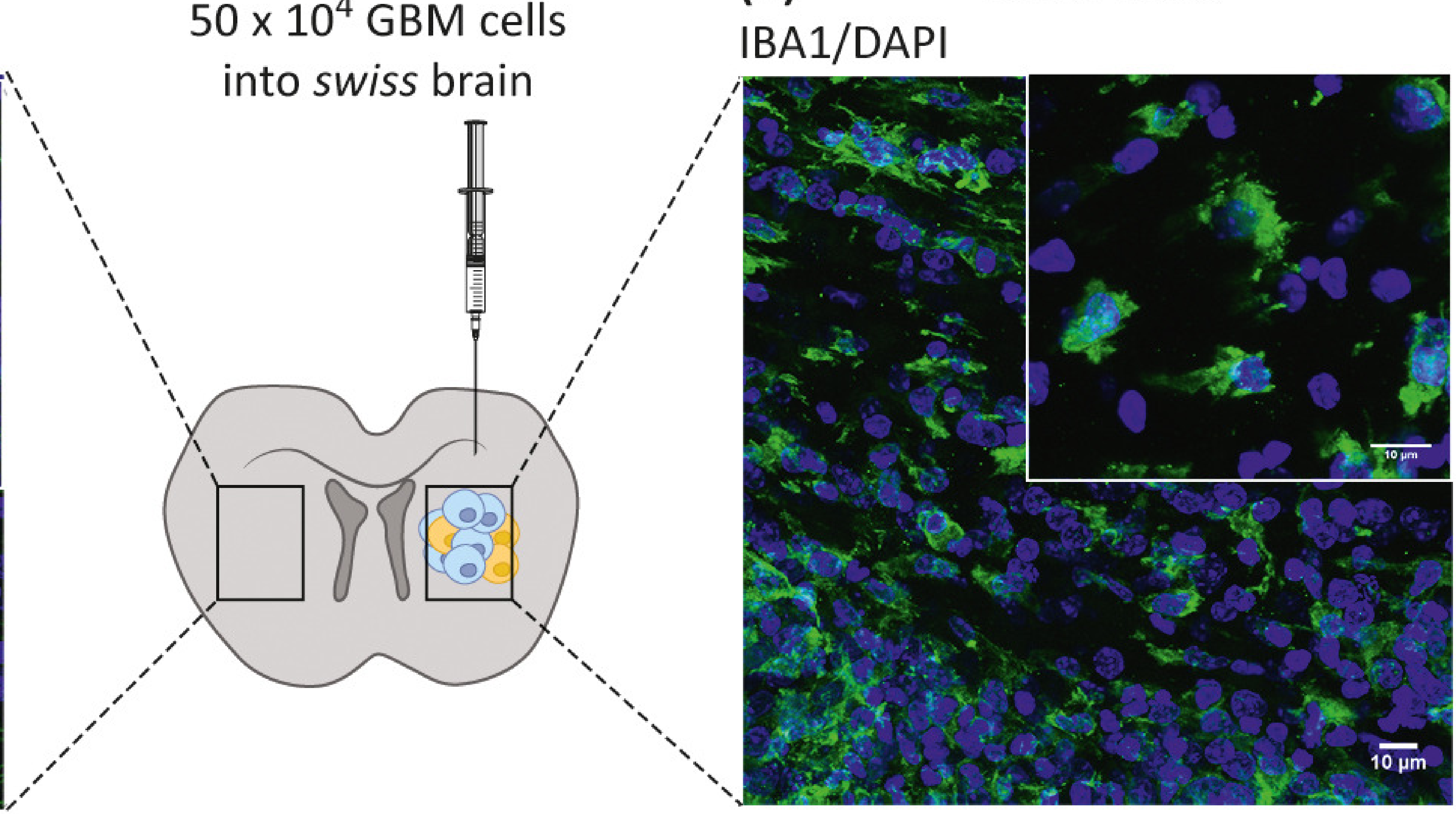

(C) "Resting" microglia

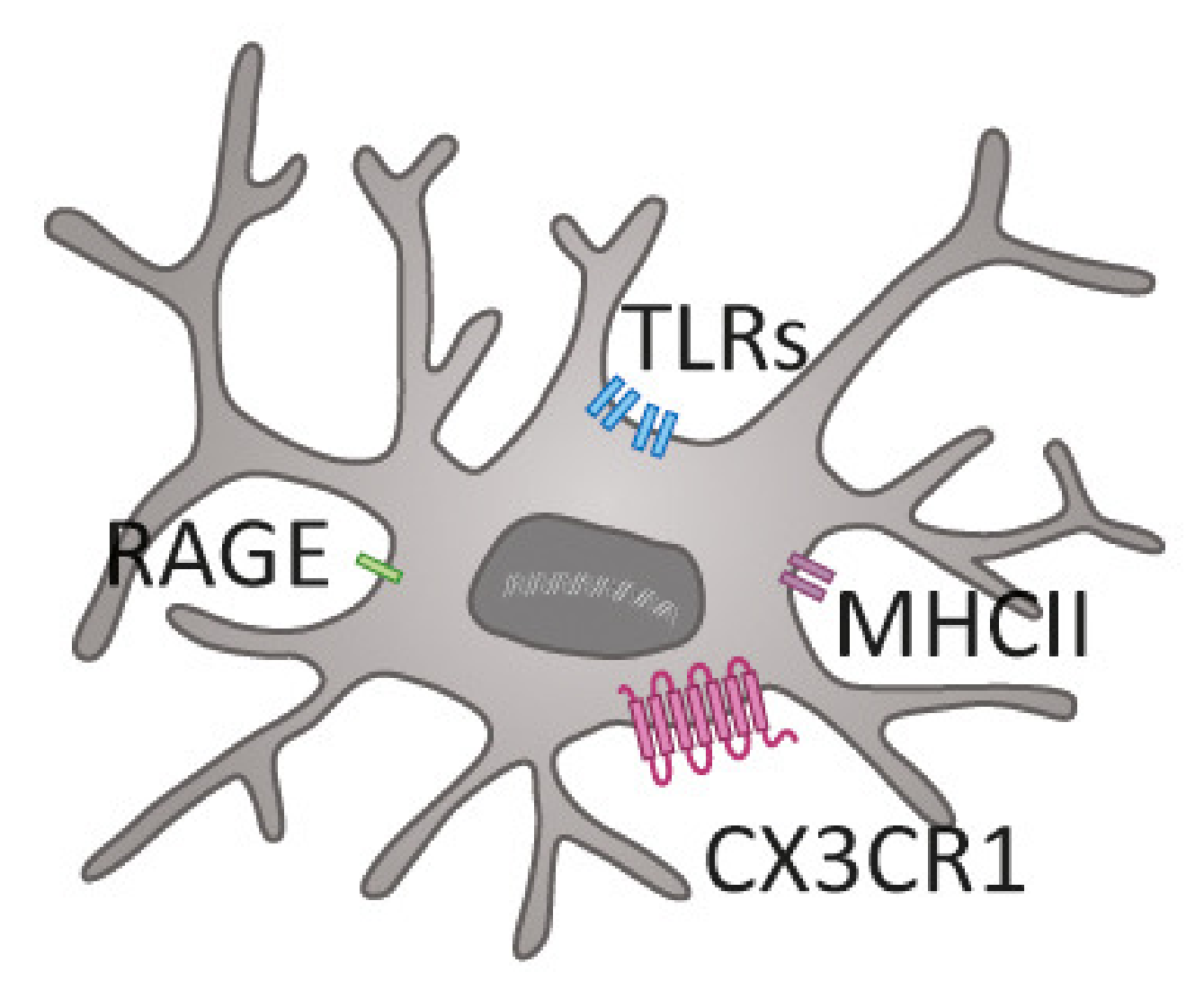

(D) Classical activation M1

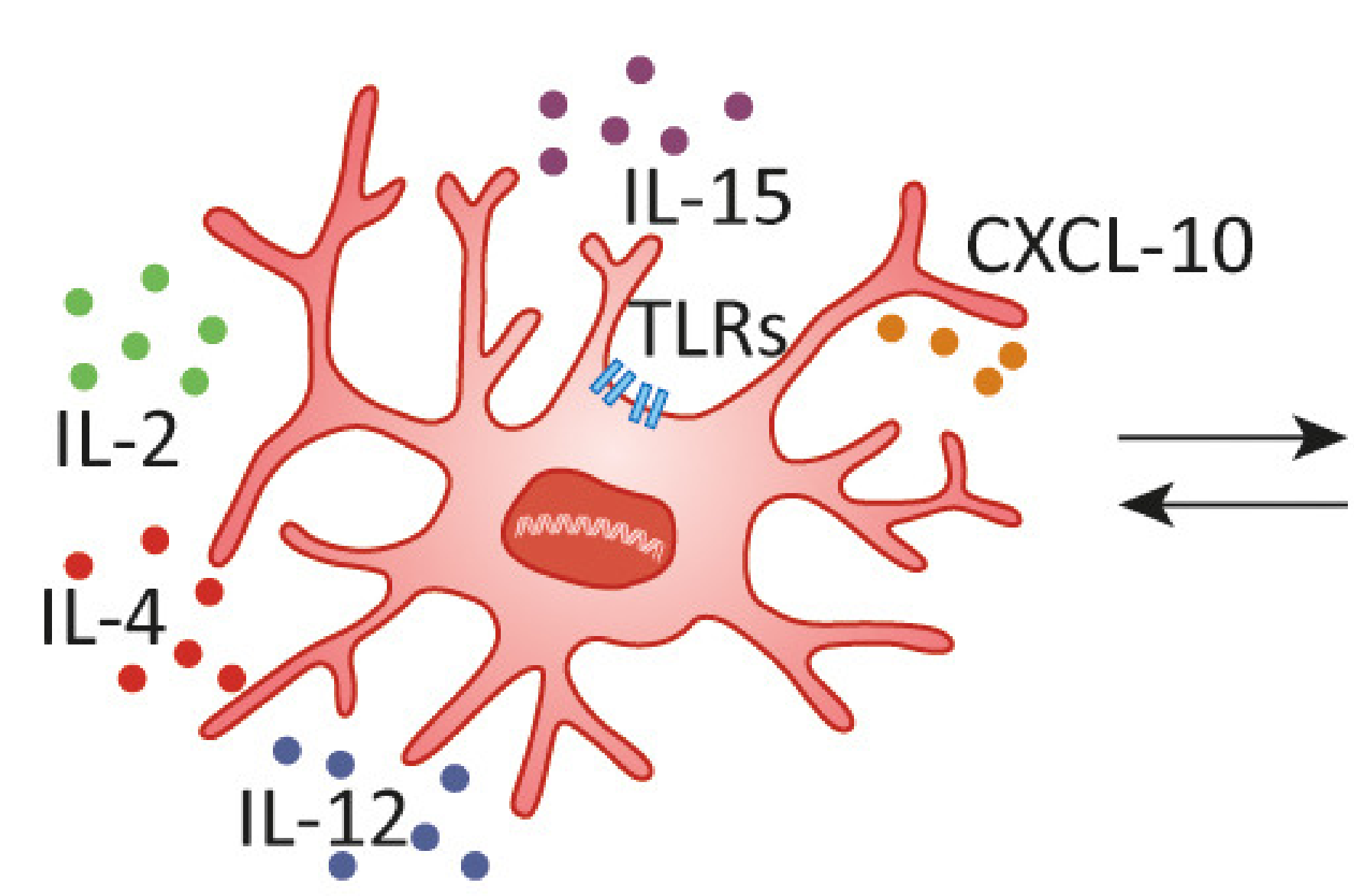

(E) Alternatively activation M2

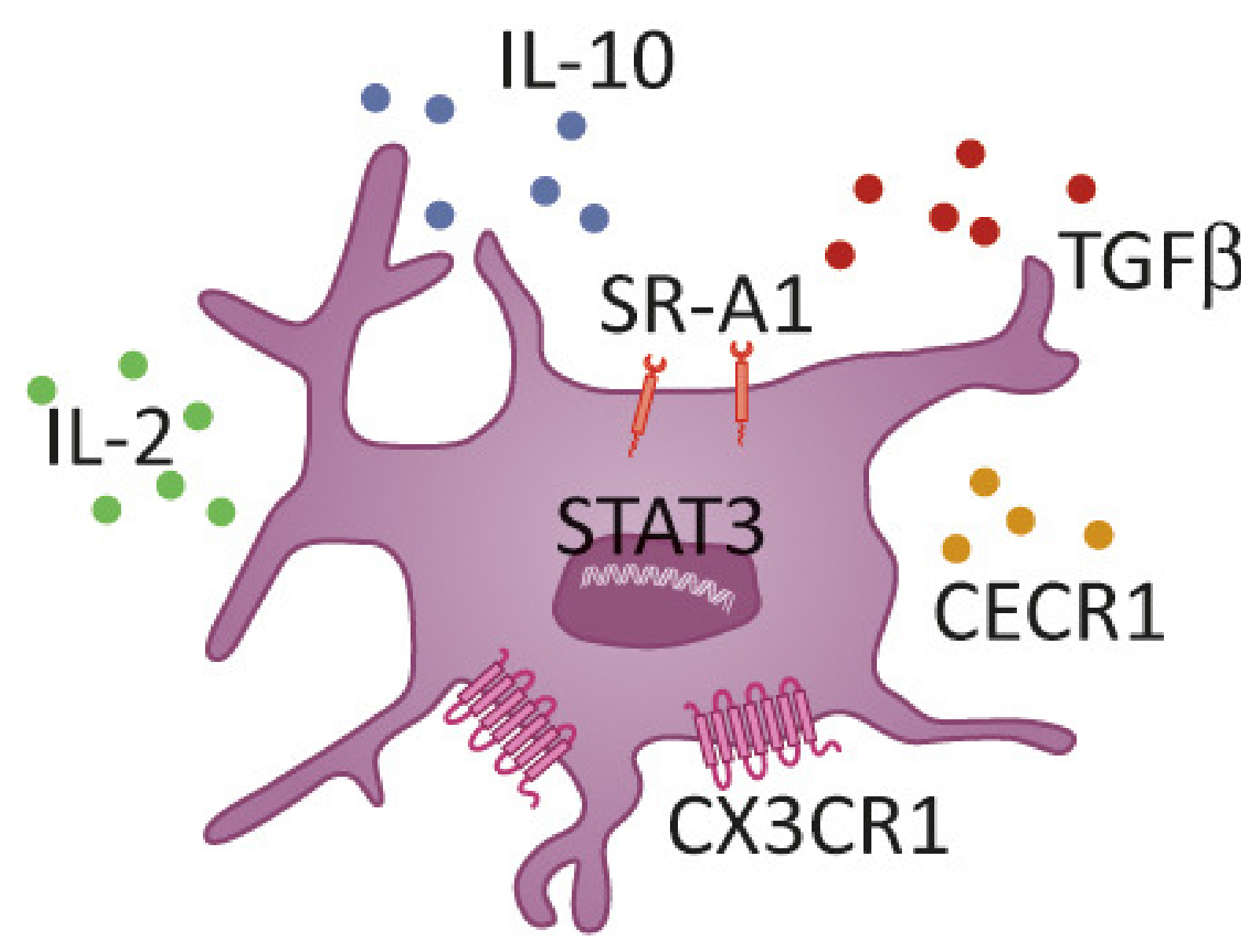

